\title{
LIMNOLOGICAL STUDY OF SERBIAN OXBOW SHAPED LAKE SREBRNO, WITH SPECIAL EMPHASIS ON THE BENTHIC COMMUNITY COMPOSITION AND STRUCTURE
}

\author{
VESNA MARTINOVIC-VITANOVIC*, SNEZANA OSTOJIC, NATASA POPOVIC, MAJA \\ RAKOVIC, VLADIMIR KALAFATIC
}

Sinisa Stankovic Institute for Biological Research, University of Belgrade, 142 Blvd. Despot Stefan, 11060 Belgrade, Serbia; e-mail: vmartino@ibiss.bg.ac.rs; vesna.m_martinovic@vektor.net

* Author for correspondence

\begin{abstract}
Martinovic-Vitanovic V., Ostojic S., Popovic N., Rakovic M., Kalafatic V.: Limnological study of Serbian oxbow shaped Lake Srebrno, with special emphasis on the benthic community composition and structure. Ekológia (Bratislava), Vol. 32, No. 1, p. 66-86, 2013.

Detailed limnological study of the Lake Srebrno (Serbia) bottom fauna was performed in March 2007. Investigations included qualitative, quantitative, and saprobiological analysis of bottom fauna communities, physical and chemical analysis of sediments and determination of chlorophyll $a$ concentration, as well as saprobic and trophic status analyses. Samples were collected at eighteen sites distributed along a shoreline and in deeper sections of the lake. Twenty taxa from nine macroinvertebrate groups were recorded. Family Chironomidae had the biggest index of participation, the highest species diversity and the largest density of populations in benthocenoses of Lake Srebrno. Faunistic similarity between sites was estimated according to Sørensen's Quotient of Similarity (QS). The majority of benthocenoses - 56\% showed a medium level of faunistic similarity (QS $=21-60 \%$ ), and $42 \%$ of benthocenoses showed a high level of faunistic similarity (QS $=61-100 \%)$. The classification of Lake Srebrno based on saprobic and trophic levels was assessed. Saprobic level was in the range from alpha-meso- to poly-alpha-mesosaprobity. The water quality of the lake ranged from class III and between IV and III class. Generally, Lake Srebrno had eutrophic status with the gradation to hypertrophy.
\end{abstract}

Key words: Lake Srebrno, composition and structure of bottom fauna communities, physical and chemical sediment analyses, Chl a, water quality, ecological status.

\section{Introduction}

Reservoirs are formed by human activities for specific purposes, in order to serve as resources of water for use in areas of water scarcity or excess. Also, they are built where there are agricultural and technological reasons to have a controlled water facility also contributing to industrial development by providing sources of hydroelectric power, as well as from tourism and recreational reasons (Thornton et al., 1990; Chapman, 1997).

Lake Srebrno (Silver Lake) is an artificial lake, damned backwater of the Danube, and as an important tourist centre in Serbia has been poorly investigated in the limnological aspect. 
Preliminary study of this lake conducted in August and October 2006, included analyses of plankton and physical and chemical water characteristics, and Chl- $a$ concentration analyses, with the aim to assess trophic status of the reservoir (Martinovic-Vitanovic, Kalafatic, 2006). Detailed limnological study of the Lake Srebrno performed in March 2007, with special emphasis on the benthic community, deal with the presence of bottom fauna species, their life cycles and relations between other members of the ecosystem having been the subject of previous studies of stagnant waters, lowland lakes formed in large river valleys: Obedska Bara marsh and Sava Lake (Jankovic, 1967, 1974, 1982; Jakovcev, 1989; Martinovic-Vitanovic, 1996; Martinovic-Vitanovic, Kalafatic, 1999; Martinovic-Vitanovic et al., 2006, 2008, 2009/2010) in Serbia, and Lake Jarun in Croatia (Kerovec et al., 1989).

Human-induced impacts on freshwater ecosystems have been increasing worldwide. Their assessment is a subject of many investigations usually based on monitoring data and analyses of abiotic and biotic factors (Kagalou et al., 2006). Freshwater benthic communities have been used as bioindicators for monitoring pollution and anthropogenic influences. Macroinvertebrates play a key role in freshwater ecosystems in linking primary production, allochthonous input and top predators (Chapman, 1997). Organisms of the bottom fauna as very important component of aquatic ecosystems are vulnerable to environmental changes; therefore, composition and structure of macrozoobenthic communities are used as indicators of aquatic ecosystem status, and in evaluating water quality (Rosenberg, Resh, 1993). According to Rîsnoveanu, Vadineanu (2003), there is little information concerning the role of the benthic macroinvertebrates in sustaining the ecosystem productivity under different trophic conditions. Lake Srebrno is under constant anthropogenic influences because of the human activities in, and around it. Having in mind that contamination of water and sediments from toxic and hazardous substances can significantly reduce the quality of aquatic environment, in our investigations Lake Srebrno was surveyed in order to detect several pollutants (heavy metals, pesticides, polycyclic aromatic hydrocarbons, phenols and polychlorinated biphenyls) by chemical sediment analysis. With the aim to assess trace metal distribution, similar investigations were performed in other European lakes which are under anthropogenic impacts: Lake Balaton, Hungary and Lake Koronia, Macedonia, Greece (Dévai, 1990; Bobori, 1996; Nguyen et al., 2005), as well as in four lakes in Quebec, Canada (Laliberte, Tremblay, 2002).

Species composition, their occurrence frequencies in benthic communities as well as quantitative aspect of assemblages are used as basis for the characterisation and assessment of the freshwater body ecological status (Chapman, 1997). Representatives of the groups Chironomidae, Chaoboridae and Oligochaeta found in Lake Srebrno bottom fauna (dominant in bottom fauna of the Obedska Bara marsh, according to Jankovic, 1967) are important indicators for pollution level and trophy of continental waters (Kerovec et al., 1989; Dévai, 1990; Specziar, Biro, 1998; Timm, 1999; Moog, 2002).

This study represents first limnological data dealing with bottom fauna composition and structure of the Lake Srebrno. Results of physical and chemical substrate analyses, determination of Chl- $a$ concentration, qualitative and quantitative bottom fauna analyses, and results of faunistic similarity of sites and sectors of the lake are given too, as well as results of saprobiological and trophical status analyses based on bioindicator concept. The purpose of 
this study was to determine the faunistic similarity of investigated sites and sectors in determined physical and chemical environment conditions, as well as, to estimate related water quality of the lake with application of trophic and saprobic system, i.e. to define ecological status of the Lake Srebrno as an important tourist centre in Serbia. Also, it is first attempt to define from the hydrobiological aspect the type of aquatic ecosystem to whom Lake Srebrno belongs in order to serve as the basis for the characterization and assessment of the ecological status of lakes in Serbia as lay down in the EU-Water Framework Directive (Solimini et al., 2006). The ultimate goal of this kind of investigations is to collect basic biological data pertinent to the elaboration of plans for the control of water and sediment quality, and to the preparation of the matter and energy budget of a reservoir. This is very important not only for the evaluation but also for the prediction of the changes occurring in the quality of water and sediment of an aquatic ecosystem.

\section{Material and methods}

\section{Study area}

Lake Srebrno is located in the north-eastern part of Serbia, $3 \mathrm{~km}$ from town of Veliko Gradiste (and subjacent of Lipovaca hill $362 \mathrm{~m}$ a.s.l.), at the Serbian-Romanian border formed by the river Danube close to the lake.

According to Martinovic-Vitanovic, Kalafatic (2002) and Martinovic-Vitanovic et al. (2006) the Serbian Danube stretch is divided into three parts:

I. Upper and lower part - Riverine zone I (upstream river part) and Riverine zone II (downstream river part): from r-km 1425 (Batina-Bezdan) to r-km 1250 (Novi Sad); and from r-km 863.4 (Iron Gate II dam) to r-km 845.6 (mouth of the Timok river into the Danube);

II. Middle part - Transitional zone (flow-through reservoir): from r-km 1216 (Slankamen) to r-km 1072 (Ram);

III. Lower part - Lacustrine zone (lake part - reservoirs: Iron Gate I and II): from r-km 1072 (Ram) to dam Iron Gate I (Djerdap I) at r-km 942.9; and from r-km 942.9 to 863.4 dam Iron Gate II (Djerdap II), respectively.

The lake is situated in the lower part of the Serbian Danube stretch, in the Lacustrine zone: lake part - Iron Gate I reservoir. Lake Srebrno is an armlet at the right-hand side of the river Danube (1069-1062 Danube r-km), separated from the Danube by upstream and downstream barriers constructed in 1971. Before reservoir formation, the Danube flooded lowland, and "fluviatile-river lake" was formed by running water actions. In Limnology, this type of lake is known as "oxbow lake" formed in flooded lowlands - "flood plain lakes" (two of the kind mentioned, Obedska Bara marsh and Lake Srebrno being in Serbia - Martinovic-Vitanovic, 1996; Martinovic-Vitanovic, Kalafatic, 1995, 1999). The lake is $14 \mathrm{~km}$ long, with an average width of $300 \mathrm{~m}$, and total area of $4 \mathrm{~km}^{2}$. Its elevation is $70 \mathrm{~m}$ a.s.l., with a maximum depth of $8 \mathrm{~m}$. Three villages are located on the lake banks: Ostrovo (eastern part), Zatonje (western part) and Kisiljevo (on the most southern part of the meander). The lake, supplied with Danube water by underground natural filtration throughout the length of sand drifts, also receives water directly from the Kisiljevo stream and canal near Ostrovo village. The north-eastern part of Serbia where the Lake Srebrno is situated is characterized by a moderate continental climate with expressed annual seasons. Winters are sharp with frequent and intense south-eastern wind ("Kosava"), and summers are very hot with high air and water temperatures.

With a hotel, a few restaurants, an auto camp, and weekend cottages Lake Srebrno represents an important tourist centre in Serbia as a multipurpose recreation centre for the town Veliko Gradiste (swimming and other body contact recreation, boating, paddling, sailing and other aesthetic recreational uses, as well as sport fishing).

\section{Site classification, field sampling and data collection}

Samples were collected in March 2007 from 18 sites, grouped in four sectors (Fig. 1). Partition of the lake into four sectors is based on the hypothesis of different types of pollution. Namely, pollution comes from tourist activities, due to the residence of a large number of tourists in the tourist centre (Sector I), and as input of pollutants from 


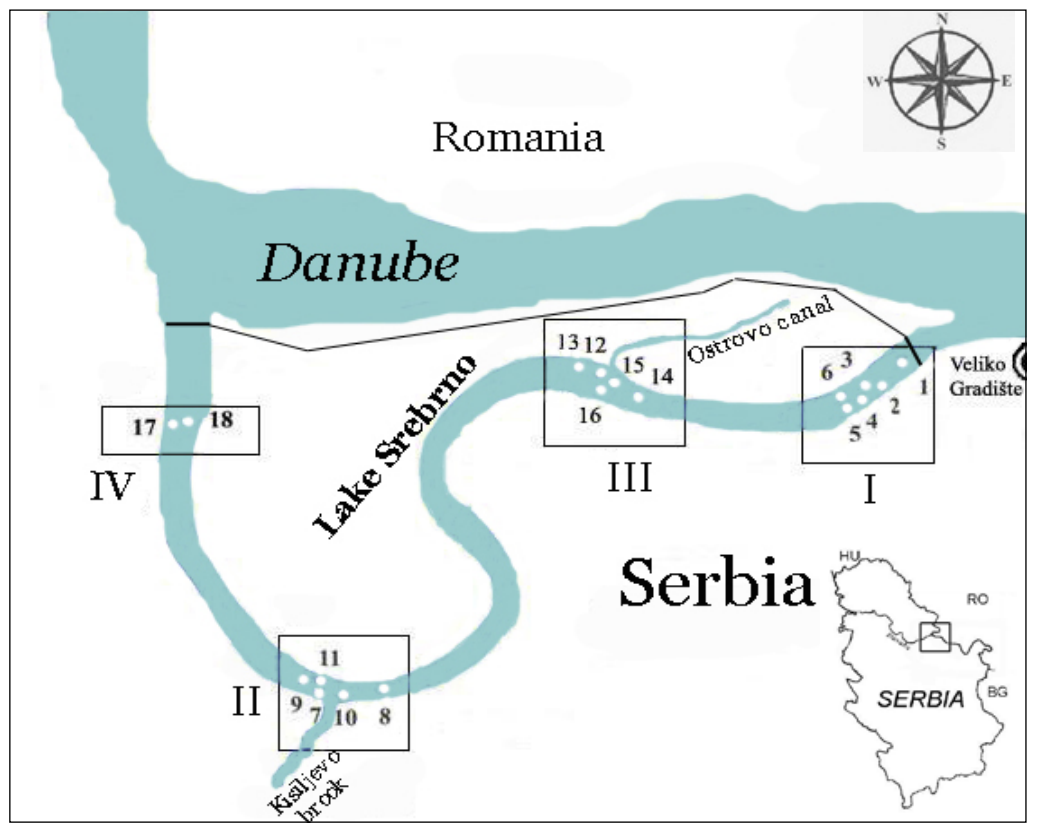

Fig. 1. Map of the Lake Srebrno with sampling sites grouped per sectors GPS positions:

Sector I - tourist centre (sites 1-6) $44^{\circ} 45^{\prime} 47.98^{\prime \prime} \mathrm{N}, 21^{0} 28^{\prime} 35.97^{\prime \prime} \mathrm{E}$

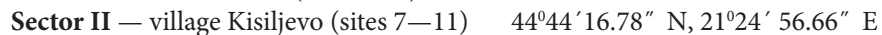

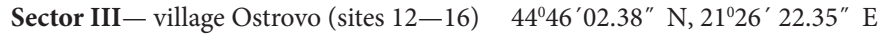

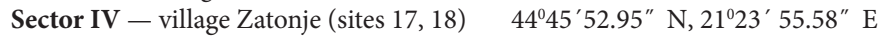

villages: Zatonje with its auto camp (IV), Ostrovo with the canal (III) and Kisiljevo with the Kisiljevo brook (II), where pollution would also come from agricultural production (crop and stock farming).

At all sampling sites, a Van Veen type of grab $\left(270 \mathrm{~cm}^{2}\right.$ grab area) was used for collecting the samples for quantitative analysis of bottom fauna, except at site 12 where a qualitative sample was taken. Substrate classification was performed by visual evaluation in situ, and in the laboratory (using stereo zoom microscope with binocular magnifier-magnification 5-50x, Krüss, Germany), based on the diameter of sediment particles (Wentworth, 1922) and according to national classification after Martinovic-Vitanovic and Kalafatic (Martinovic-Vitanovic, Kalafatic, 1995; Lakusic et al., 2005).

Aquatic invertebrates in each sample were separated from the sediment by washing and sieving (mesh size 200 $\mu \mathrm{m})$. All samples were fixed in situ with $4 \%$ formaldehyde and then transported to the laboratory for further processing. A stereo zoom microscope with binocular magnifier (magnification 5-50x), Krüss, Germany, and microscope (10x10, and 10x40), Opton, Germany were used for sorting and identification of organisms. Appropriate keys were used for determining bottom fauna representatives either up to the species level or to the lowest possible taxonomic level: Brinkhurst, Jamieson (1971); Elliot, Mann (1979); Timm (1999); Wiederholm (1983); Lellak (1980) and Croft (1986).

Physical and chemical sediment analyses were performed using standard methods and techniques, APHA-AWWA-WEF (1995), in the Institute of Public Health, Belgrade. The following parameters were analyzed: $\mathrm{pH}$, total nitrogen, total phosphorus, total organic carbon (TOC), heavy metals (As, $\mathrm{Cu}, \mathrm{Zn}, \mathrm{Cr}, \mathrm{Cd}, \mathrm{Ni}, \mathrm{Pb}$ and $\mathrm{Hg}$ ), and pollutants: cyanides, pesticides, polycyclic aromatic hydrocarbons (PAHs), polychlorinated biphenyls (PCBs) and phenols. Canadian and American Standards (CCME and USEPA) - Sediment Quality Guidelines (CCME, 2002; USEPA, 2002) are used to present results of silt analyses. 


\section{Data analysis}

The trophical status of the lake was determined according to bottom fauna production (absolute abundance), algal production by analysis of chlorophyll a concentration in the lake water and by measuring water transparency with a Secchi disc (OECD, 1982). Relative algal biomass in samples was estimated as concentration of Chl- $a$ (maximum concentration of Chl- $a$ as a criteria; OECD, 1982), measured spectrophotometrically from a hot $90 \%$ ethanol extract (APHA-AWWA-WEF, 1995).

Spatial distribution of benthocenoses of Lake Srebrno was analyzed. Appearance frequencies $(\mathrm{F}=0-1)$ of taxa and groups recorded at the sampling sites ( $1-18$ sites and I-IV sectors) were calculated as $\mathbf{F}=\mathbf{m} \cdot \mathbf{M}^{-1}$, where $\mathbf{m}$ stands for the number of samples, in which the particular species was found, and $\mathbf{M}$ stands for the total number of samples. Correspondence Analysis (CA) was used to estimate the differences between sampling sites and between sectors of Lake Srebrno based on presence/absence of macroinvertebrate species. The analyses were carried out using the computer package STATISTICA (StatSoft Inc. 1997). Similarities/dissimilarities in regard to qualitative composition of benthocenosis were calculated according to the Sørensen Quotient of Similarity (QS) at all sampling sites (Sørensen, 1948), given in percent, indicating the similarity or dissimilarity between sites with respect to composition of the macrozoobenthic assemblages. For the calculation of Sørensen QS the following formula was used: QS = 2c/a+b.100, where $\mathbf{c}$ stands for the number of species in common between two locations, a stands for the total number of species at one location, and $\mathbf{b}$ stands for the total number of species at the other location.

Density of the bottom fauna populations at sampling sites was defined by counting individuals in quantitative samples and calculated per unit area of the bottom surface (APHA-AWWA-WEF, 1995). Absolute abundance of organisms is given as the number of individuals per square meter - no ind. $\mathrm{m}^{-2}$, and the participation of every benthic group in the total abundance of benthocenosis at sampling sites is given as a percentage.

The Saprobic index S, according to Pantle, Buck (1955) and classification of saprobity levels, according to Moog (2002), were used to estimate the water quality at each site. For the calculation of $S$ the following formula was used: $\mathbf{S}=\mathbf{\Sigma} \mathbf{s h} / \mathbf{\Sigma} \mathbf{h}$; where $\mathbf{s}$ stands for saprobic valence i.e. species degree of saprobity (after Moog ed., 1995; 2002), and $\mathbf{h}$ for abundance (rare $=1$, frequent $=3$, abundant $=5$ ). Water quality was also estimated based on criteria set out in official legislation (Official gazette of the SFRY, 1978; Official gazette of the SRS, 1968), according to this accumulation should have the same water quality as a main watercourse. The following scheme (Martinovic-Vitanovic et al., 2008) shows the classification of the Saprobic index S with the water quality classes and the saprobity versus trophy levels:

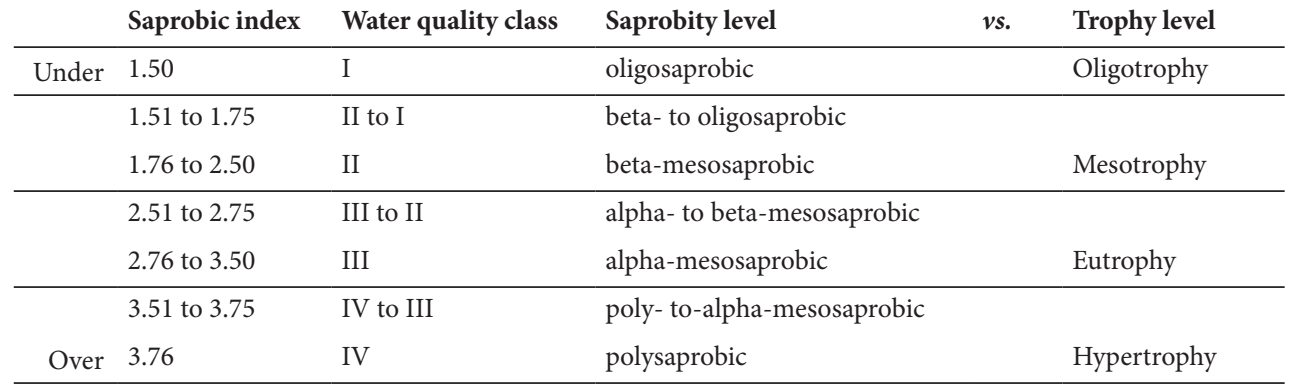

\section{Results}

\section{Analyses of environmental characteristics}

Table 1 summarizes morphometric characteristics of habitats, physical parameters of sediments and water, and Lake Srebrno Chl-a concentration.

Average depth of the whole lake and per sectors was around $5 \mathrm{~m}$, and average water temperature was around $10{ }^{\circ} \mathrm{C}$. Water transparency was low and relatively uniform, ranged from 0.80 (site 1) to $1.40 \mathrm{~m}$ (site 13). Chlorophyll $a$ average sector concentrations were between $23.86 \mu \mathrm{g} / \mathrm{l}$ (Sector III) and $42.92 \mu \mathrm{g} / \mathrm{l}$ (Sector IV). 
$\mathrm{T}$ a b l e 1. Lake Srebrno sediment and water physical characteristics and chlorophyll a concentration (single and mean values per sector), in March 2007.

\begin{tabular}{|c|c|c|c|c|c|c|}
\hline Sector & Sampling site & $\begin{array}{l}\text { Water temperature } \\
\left({ }^{\circ} \mathrm{C}\right)\end{array}$ & $\begin{array}{l}\text { Depth } \\
(\mathrm{m})\end{array}$ & $\begin{array}{l}\text { Transparency } \\
\text { (m) }\end{array}$ & $\begin{array}{l}\text { Chlorophyll } a \\
\text { concentration } \\
(\mu \mathrm{g} / \mathrm{l})\end{array}$ & Substrate \\
\hline \multirow{7}{*}{ I } & 1 & 10.00 & 5.00 & 0.80 & 22.94 & l, vfs, d \\
\hline & 2 & 10.20 & 5.00 & 1.20 & 22.20 & $1, \mathrm{cs}$ \\
\hline & 3 & 10.20 & 5.50 & 1.20 & 19.98 & $1, \mathrm{~ms}$ \\
\hline & 4 & 10.20 & 5.50 & 1.15 & 22.20 & l, vfs, d \\
\hline & 5 & 10.20 & 5.20 & 1.20 & 26.64 & $1, \mathrm{fs}$ \\
\hline & 6 & 10.20 & 5.50 & 1.15 & 30.34 & $1, \mathrm{cs}, \mathrm{csd}, \mathrm{p}$ \\
\hline & $\tilde{\mathbf{n}}$ & 10.17 & 5.28 & 1.12 & 24.05 & \\
\hline \multirow{6}{*}{ II } & 7 & 11.00 & 2.50 & 0.90 & 31.08 & vfs, fsd, d \\
\hline & 8 & 12.00 & 7.00 & 1.10 & 28.12 & $1, \mathrm{cs}, \mathrm{d}$ \\
\hline & 9 & 11.00 & 4.00 & 0.90 & 25.90 & $\mathrm{~ms}, \mathrm{msd}$ \\
\hline & 10 & 11.00 & 5.50 & 1.10 & 25.16 & $1, \mathrm{~ms}$ \\
\hline & 11 & 11.20 & 6.00 & 1.10 & 26.64 & $\mathrm{~ms}, \mathrm{msd}$ \\
\hline & $\tilde{\mathbf{n}}$ & 11.24 & 5.00 & 1.02 & 27.38 & \\
\hline \multirow{6}{*}{ III } & 12 & 10.20 & 3.00 & 1.00 & - & fs, fsd \\
\hline & 13 & 10.20 & 4.50 & 1.40 & 18.50 & vfs, d \\
\hline & 14 & 9.80 & 4.50 & 1.15 & 25.90 & fs \\
\hline & 15 & 9.20 & 6.00 & 1.10 & 22.20 & vfs, d \\
\hline & 16 & 9.20 & 7.00 & 1.15 & 28.86 & vfs \\
\hline & $\tilde{\mathbf{n}}$ & 9.72 & 5.00 & 1.16 & 23.86 & \\
\hline \multirow{3}{*}{ IV } & 17 & 11.00 & 5.50 & 1.20 & 31.08 & vfs, d \\
\hline & 18 & 10.80 & 5.50 & 1.00 & 54.76 & $1, \mathrm{~ms}$ \\
\hline & $\tilde{\mathbf{n}}$ & 10.90 & 5.50 & 1.10 & 42.92 & \\
\hline
\end{tabular}

Substrate classification according to Wentworth (1922): p - pebble (4-64 mm); csd - coarse sand $(500 \mu \mathrm{m}-1 \mathrm{~mm})$; msd - medium sand $(250-500 \mu \mathrm{m})$; fsd - fine sand $(125-250 \mu \mathrm{m})$; cs - coarse silt $(31-63 \mu \mathrm{m})$; ms — medium silt $(15.6-31 \mu \mathrm{m})$; fs - fine silt $(7.8-15.6 \mu \mathrm{m})$; vfs - very fine silt $(3.9-7.8 \mu \mathrm{m}) ; 1$ - loess; $\mathrm{d}$ - detritus.

Substrate at 18 sampling sites was composed of loess, silt, silt with detritus, sand and pebble. Silt of different particle sizes was present at all 18 sites; detritus was present at all four sectors (39\% of sites); loess was present at $50 \%$ of sites (sectors I, II and IV); at $28 \%$ of sites (I, II and III) was recorded presence of sand; and pebble was present only at site 6 (sector I).

Table 2 summarizes the results of sediment chemical analysis. Regarding to silt $\mathrm{pH}$ values substrate was medium to weakly alkaline. Total nitrogen concentrations were under $0.7 \%$, while total phosphorus concentrations were between $429.4 \mathrm{mg} / \mathrm{kg}$ (site 14) and 1, $172.5 \mathrm{mg}$ / $\mathrm{kg}$ (site 9). The highest percent of total organic carbon (TOC) was $4.37 \%$ (site 8 ). Canadian and American Guidelines for assessment of contaminated sediments in freshwater ecosystems did not provide standards for total $\mathrm{N}$, total $\mathrm{P}$ and TOC as water eutrophication factors (CCME, 2002; USEPA, 2002). Cyanide concentrations were under the detection limit $(<1.0$ $\mathrm{mg} / \mathrm{kg}$ ), as well as mercury concentrations $(<0.6 \mathrm{mg} / \mathrm{kg})$. Other heavy metals (As, Cu, Zn, $\mathrm{Cr}, \mathrm{Cd}, \mathrm{Ni}, \mathrm{Pb}$ ) were present at all sites in narrow range of variations (Table 2 ). At several sites 


\begin{tabular}{|c|c|c|c|c|c|c|c|c|c|c|c|c|c|c|c|c|c|c|}
\hline 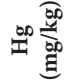 & $\begin{array}{l}\stackrel{b}{\dot{0}} \\
v\end{array}$ & $\begin{array}{l}0 \\
\stackrel{0}{0} \\
v\end{array}$ & $\begin{array}{l}0 \\
\stackrel{0}{v} \\
v\end{array}$ & $\begin{array}{l}\text { O̊ } \\
\text { V }\end{array}$ & $\begin{array}{l}\text { : } \\
\dot{v}\end{array}$ & $\begin{array}{l}\stackrel{0}{0} \\
\dot{v}\end{array}$ & $\begin{array}{l}\dot{0} \\
\dot{\mathrm{v}}\end{array}$ & $\begin{array}{l}\text { : } \\
\text { v }\end{array}$ & $\begin{array}{l}\text { : } \\
\dot{v}\end{array}$ & $\begin{array}{l}\stackrel{0}{0} \\
\dot{v}\end{array}$ & $\begin{array}{l}\stackrel{0}{0} \\
\text { v }\end{array}$ & $\begin{array}{l}\dot{b} \\
\dot{v}\end{array}$ & $\begin{array}{l}\dot{0} \\
\dot{v}\end{array}$ & $\begin{array}{l}\dot{D} \\
v\end{array}$ & $\begin{array}{l}\stackrel{0}{0} \\
\dot{v}\end{array}$ & $\begin{array}{l}\dot{b} \\
\dot{v}\end{array}$ & $\begin{array}{l}\stackrel{0}{0} \\
\stackrel{v}{v}\end{array}$ & $\begin{array}{l}\stackrel{0}{0} \\
\stackrel{v}{v}\end{array}$ \\
\hline 离 & 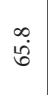 & $\vec{n}$ & $\begin{array}{l}\text { In } \\
\text { to }\end{array}$ & $\begin{array}{l}n \\
\infty \\
\infty \\
\infty\end{array}$ & $\begin{array}{l}\infty \\
\stackrel{+}{0}\end{array}$ & $\hat{\vec{b}}$ & $\begin{array}{l}\text { İ } \\
\text { in }\end{array}$ & $\begin{array}{l}\infty \\
\stackrel{+}{m}\end{array}$ & 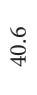 & $\stackrel{m}{m}$ & $\stackrel{\text { ते }}{\text { in }}$ & $\stackrel{b}{\stackrel{ن}{m}}$ & $\stackrel{\text { Iे }}{\mathrm{N}}$ & $\begin{array}{l}\stackrel{b}{0} \\
\stackrel{f}{+}\end{array}$ & $\stackrel{n}{m}$ & त. & $\begin{array}{l}\text { ñ } \\
\qquad\end{array}$ & $\vec{m}$ \\
\hline 乙 & ָூ & $\overrightarrow{\vec{b}}$ & $\underset{\mathrm{N}}{\mathrm{N}}$ & $\begin{array}{l}\infty \\
\dot{\infty} \\
\infty\end{array}$ & $\overrightarrow{\substack{\infty \\
\infty}}$ & $\overrightarrow{\mathrm{N}}$ & $\underset{\tilde{m}}{\tilde{m}}$ & હొ & तुં & $\begin{array}{l}\stackrel{0}{a} \\
\end{array}$ & $\begin{array}{l}n \\
\infty \\
\infty \\
\infty\end{array}$ & 苟 & 品 & ָુ & 字 & $\begin{array}{l}0 \\
\text { ¿ें }\end{array}$ & $\hat{\ddot{q}}$ & $\begin{array}{l}\circ \\
\dot{\sigma}\end{array}$ \\
\hline 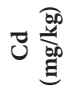 & $\stackrel{\circ}{\circ}$ & ?ֶ? & $\hat{o}$ & $\stackrel{o}{o}$ & $\stackrel{\infty}{0}$ & $\stackrel{0}{\circ}$ & กี & t: & ?ִ & $\stackrel{m}{o}$ & กี & ?3. & ơ & ?n & ชี & ?. & $\stackrel{0}{\circ}$ & กี \\
\hline 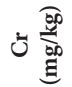 & $\stackrel{\circ}{\dot{q}}$ & $\stackrel{\rightleftarrows}{\Rightarrow}$ & 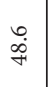 & $\stackrel{+}{\stackrel{n}{n}}$ & iे & $\hat{\mathrm{in}}$ & ֶु & 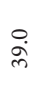 & $\begin{array}{l}\stackrel{0}{+} \\
\stackrel{+}{+}\end{array}$ & $\stackrel{n}{a}$ & $\begin{array}{l}\text { mb } \\
\stackrel{\sim}{\sim}\end{array}$ & $\underset{\sim}{\stackrel{n}{\pi}}$ & 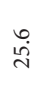 & 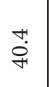 & تั. & 㝋 & 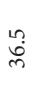 & 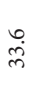 \\
\hline 空 & $\begin{array}{l}\text { } \\
\stackrel{0}{\circ}\end{array}$ & $\ddot{\leftrightarrow}$ & $\begin{array}{l}\text { +் } \\
\stackrel{+}{\sigma}\end{array}$ & 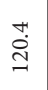 & 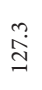 & $\stackrel{\infty}{\stackrel{I}{\exists}}$ & $\stackrel{\infty}{\tilde{n}}$ & $\stackrel{i}{i}$ & $\stackrel{\infty}{\infty}$ & $\stackrel{m}{i}$ & 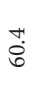 & ?̊. & $\begin{array}{l}\stackrel{n}{\mathfrak{H}} \\
\stackrel{f}{1}\end{array}$ & $\begin{array}{l}0 \\
\text { i. } \\
\infty\end{array}$ & $\stackrel{m}{i}$ & $\stackrel{\partial}{\dot{f}}$ & $\stackrel{\circ}{\circ}$ & $\overrightarrow{8}$ \\
\hline 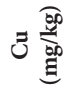 & $\stackrel{\infty}{\sim}$ & $\stackrel{\circ}{\stackrel{m}{m}}$ & 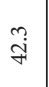 & $\stackrel{m}{\vec{F}}$ & $\stackrel{\infty}{a}$ & $\stackrel{n}{n}$ & $\stackrel{\circ}{\infty}$ & $\stackrel{\vec{m}}{m}$ & ্ֻলি & ढें & 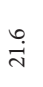 & $\stackrel{+}{\stackrel{+}{+}}$ & $\overrightarrow{\stackrel{N}{d}}$ & $\stackrel{m}{m}$ & & $\stackrel{n}{8}$ & तु & $\stackrel{\infty}{\sim}$ \\
\hline 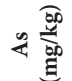 & $\stackrel{g}{\mathrm{I}}$ & $\stackrel{\infty}{\subseteq}$ & 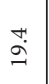 & 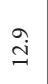 & बें & $\stackrel{\hat{n}}{n}$ & $\stackrel{0}{\circ}$ & $\overrightarrow{\text { ji }}$ & $\stackrel{f}{+}$ & $\overrightarrow{\mathrm{I}}$ & $\vec{\infty}$ & $\stackrel{N}{=}$ & $\stackrel{n}{\infty}$ & $\stackrel{\circ}{\varrho}$ & ${ }_{\infty}^{\circ}$ & ڤે & $\begin{array}{l}6 \\
\stackrel{I}{I}\end{array}$ & $\stackrel{m}{\varrho}$ \\
\hline 解 & $\begin{array}{l}\stackrel{0}{\text { D. }} \\
\text { v }\end{array}$ & 㝘 & బू. & $\begin{array}{l}\stackrel{\dot{\Xi}}{\mathrm{v}} \\
\mathrm{v}\end{array}$ & $\begin{array}{l}\stackrel{\dot{\Xi}}{\mathrm{v}} \\
\mathrm{v}\end{array}$ & $\stackrel{O}{\stackrel{0}{\vec{v}}}$ & 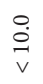 & $\begin{array}{l}\stackrel{0}{\stackrel{े}{v}} \\
\mathrm{v}\end{array}$ & $\begin{array}{l}\stackrel{0}{\grave{v}} \\
\mathrm{v}\end{array}$ & $\begin{array}{l}\stackrel{0}{\mathrm{v}} \\
\mathrm{v}\end{array}$ & $\begin{array}{l}\stackrel{\circlearrowright}{\text { Dे }} \\
\text { v }\end{array}$ & $\begin{array}{l}\stackrel{\dot{C}}{\mathrm{~V}} \\
\mathrm{v}\end{array}$ & $\begin{array}{l}\stackrel{\dot{C}}{\mathrm{v}} \\
\mathrm{v}\end{array}$ & 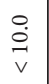 & $\begin{array}{l}\stackrel{\dot{\Xi}}{\vec{v}} \\
\mathrm{v}\end{array}$ & $\begin{array}{l}\stackrel{\dot{O}}{\mathrm{~V}} \\
\mathrm{v}\end{array}$ & 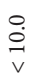 & $\begin{array}{l}\stackrel{0}{0} \\
\stackrel{\vec{v}}{v}\end{array}$ \\
\hline 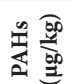 & 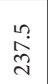 & $\begin{array}{l}\tilde{N} \\
\underset{\infty}{\sim}\end{array}$ & $\stackrel{0}{\circ}$ & $\underset{\underset{N}{*}}{\vec{N}}$ & 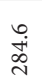 & 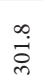 & $\stackrel{\infty}{\dot{\infty}}$ & $\begin{array}{l}\infty \\
\underset{\mathcal{J}}{ }\end{array}$ & 苟 & $\overrightarrow{\stackrel{d}{J}}$ & $\begin{array}{l}\infty \\
\infty \\
\infty \\
\infty \\
\infty\end{array}$ & 苞 & $\begin{array}{l}\dot{0} \\
\ddot{q}\end{array}$ & 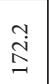 & $\stackrel{\vec{m}}{\vec{g}}$ & 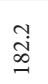 & $\begin{array}{l}\infty \\
\text { त् } \\
\text { ñ }\end{array}$ & 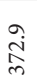 \\
\hline 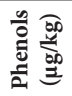 & $\begin{array}{l}\stackrel{\dot{D}}{v} \\
\vec{v}\end{array}$ & $\stackrel{\stackrel{\dot{\Xi}}{v}}{\mathrm{v}}$ & 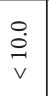 & 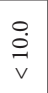 & $\begin{array}{l}\stackrel{\bigcirc}{\vec{v}} \\
\vec{v}\end{array}$ & 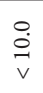 & $\stackrel{\stackrel{\leftrightarrow}{\ominus}}{\mathrm{v}}$ & $\stackrel{\stackrel{0}{\vec{v}}}{\mathrm{v}}$ & $\begin{array}{l}\stackrel{0}{\vec{v}} \\
\mathrm{v}\end{array}$ & $\begin{array}{l}\stackrel{0}{\mathrm{v}} \\
\mathrm{v}\end{array}$ & $\begin{array}{l}\stackrel{\circlearrowright}{\vec{v}} \\
\vec{v}\end{array}$ & $\stackrel{\stackrel{\dot{\Xi}}{\mathrm{v}}}{\mathrm{v}}$ & $\stackrel{\stackrel{\dot{\Xi}}{v}}{\mathrm{v}}$ & $\stackrel{\stackrel{\leftrightarrow}{\mathrm{d}}}{\mathrm{v}}$ & $\stackrel{\stackrel{\leftrightarrow}{\dot{\Delta}}}{\mathrm{v}}$ & $\stackrel{\stackrel{\leftrightarrow}{\ominus}}{\mathrm{v}}$ & $\begin{array}{l}\stackrel{O}{\stackrel{v}{v}} \\
\vec{v}\end{array}$ & $\begin{array}{l}\stackrel{\circ}{\stackrel{0}{v}} \\
\stackrel{v}{v}\end{array}$ \\
\hline 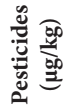 & $\stackrel{\stackrel{\leftrightarrow}{\ominus}}{\mathrm{v}}$ & $\stackrel{\stackrel{\ominus}{\vec{v}}}{\mathrm{v}}$ & $\stackrel{\stackrel{\ominus}{\vec{v}}}{\mathrm{v}}$ & 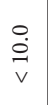 & $\stackrel{\stackrel{\dot{\Xi}}{v}}{\mathrm{v}}$ & $\stackrel{O}{\stackrel{\dot{\vec{v}}}{ }}$ & $\stackrel{\stackrel{\dot{\Xi}}{v}}{\mathrm{v}}$ & 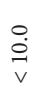 & $\stackrel{\stackrel{0}{\ominus}}{\mathrm{v}}$ & $\stackrel{\stackrel{O}{\ominus}}{v}$ & $\begin{array}{l}\stackrel{0}{\dot{D}} \\
\vec{v}\end{array}$ & $\stackrel{\stackrel{\leftrightarrow}{\vec{v}}}{\mathrm{v}}$ & $\stackrel{\stackrel{\dot{\Xi}}{\mathrm{v}}}{\mathrm{v}}$ & $\stackrel{\stackrel{\leftrightarrow}{\vec{v}}}{\mathrm{v}}$ & $\stackrel{\stackrel{\dot{\vec{v}}}{\mathrm{v}}}{ }$ & $\stackrel{\stackrel{\dot{\Xi}}{v}}{\mathrm{v}}$ & $\begin{array}{l}\stackrel{0}{\ominus} \\
\stackrel{v}{ }\end{array}$ & $\begin{array}{l}\stackrel{0}{\vec{v}} \\
\mathrm{v}\end{array}$ \\
\hline 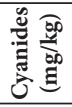 & $\stackrel{\stackrel{\rho}{\vec{v}}}{\vec{v}}$ & $\stackrel{\stackrel{\vec{v}}{\mathrm{v}}}{\mathrm{s}}$ & $\stackrel{\stackrel{\vec{v}}{\mathrm{v}}}{\mathrm{v}}$ & $\stackrel{\stackrel{\leftrightarrow}{\vec{v}}}{\mathrm{v}}$ & $\stackrel{\stackrel{\vec{v}}{\mathrm{v}}}{\mathrm{s}}$ & $\stackrel{\stackrel{\vec{I}}{v}}{ }$ & $\stackrel{\stackrel{\vec{j}}{\mathrm{v}}}{ }$ & $\stackrel{\circ}{\vec{v}}$ & $\stackrel{\stackrel{\leftrightarrow}{\vec{v}}}{\mathrm{v}}$ & $\stackrel{\circ}{\overrightarrow{\vec{v}}}$ & $\stackrel{\stackrel{\rho}{\vec{v}}}{\vec{v}}$ & $\stackrel{\stackrel{\vec{I}}{\mathrm{v}}}{\mathrm{s}}$ & $\stackrel{\stackrel{\vec{I}}{\mathrm{v}}}{ }$ & $\stackrel{\stackrel{\vec{v}}{\mathrm{v}}}{ }$ & 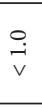 & $\stackrel{\stackrel{\vec{j}}{\mathrm{v}}}{ }$ & $\stackrel{\stackrel{\vec{v}}{v}}{\text {. }}$ & $\stackrel{\stackrel{\rho}{\vec{v}}}{\vec{v}}$ \\
\hline$\stackrel{a}{\circ}$ & 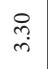 & 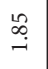 & $\stackrel{\text { İ }}{\mathrm{i}}$ & $\stackrel{\circ}{\circ}$ & 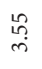 & $\stackrel{\mathscr{L}}{\infty}$ & $\underset{i}{\stackrel{\sim}{i}}$ & $\stackrel{\text { m}}{\vec{f}}$ & $\vec{m}$ & $\stackrel{\overbrace{}}{i}$ & 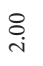 & $\stackrel{\leftrightarrow}{\stackrel{n}{-}}$ & $\stackrel{\infty}{\sim}$ & f & $\underset{\mathrm{i}}{\overrightarrow{\mathrm{H}}}$ & ڤ̊ & $\stackrel{\leftrightarrow}{\stackrel{\leftrightarrow}{\circ}}$ & 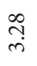 \\
\hline 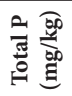 & $\begin{array}{c}\infty \\
\stackrel{+}{+} \\
i \\
i n\end{array}$ & ते & 集 & ณึ่ & હે్ٍతి & $\begin{array}{l}\ddot{0} \\
0 \\
0\end{array}$ & \begin{tabular}{l}
$\infty$ \\
\multirow{1}{0}{} \\
$\hat{b}$
\end{tabular} & $\begin{array}{l}\text { ते } \\
\text { a }\end{array}$ & $\stackrel{\substack{n \\
\Xi}}{\Xi}$ & 葡 & î & 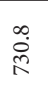 & 员 & 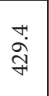 & $\begin{array}{l}\text { oे } \\
\text { in }\end{array}$ & 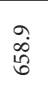 & 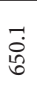 & ふุँ \\
\hline 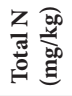 & ঙ̆ & તิ & 苗 & $\stackrel{\mathscr{m}}{\tilde{\sigma}}$ & ఫे & $\stackrel{\text { ?ִ }}{0}$ & ֻٕ: & in? & f? & ल. & กิ่ & 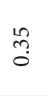 & $\stackrel{\infty}{\overbrace{0}^{\infty}}$ & $\stackrel{\text { న్ }}{0}$ & ले & : & F. & ֻु. \\
\hline t & $\underset{\infty}{\stackrel{H}{*}}$ & 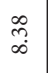 & $\stackrel{\substack{n \\
\infty}}{\infty}$ & $\begin{array}{l}\stackrel{2}{0} \\
\infty \\
\infty\end{array}$ & $\begin{array}{l}\infty \\
\infty \\
\infty \\
\infty\end{array}$ & $\vec{\infty}$ & $\underset{\infty}{\stackrel{\infty}{\infty}}$ & $\underset{\infty}{\approx}$ & $\underset{\infty}{\text { ने }}$ & సે & $\begin{array}{l}9 \\
\infty \\
\infty\end{array}$ & $\underset{\infty}{\stackrel{\infty}{\infty}}$ & $\underset{\infty}{\stackrel{\infty}{\infty}}$ & $\begin{array}{l}\vec{\sigma} \\
\vec{\infty}\end{array}$ & $\underset{\infty}{\infty}$ & $\begin{array}{l}\stackrel{0}{\infty} \\
\infty\end{array}$ & $\stackrel{\circ}{\infty}$ & $\underset{\infty}{\stackrel{9}{0}}$ \\
\hline 苛 & $\neg$ & $\sim$ & $m$ & $r$ & in & 6 & $n$ & $\infty$ & $a$ & 은 & $=$ & $\simeq$ & $\stackrel{m}{2}$ & \pm & $\stackrel{2}{2}$ & $\stackrel{0}{-}$ & 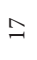 & $\stackrel{\infty}{\rightarrow}$ \\
\hline
\end{tabular}


concentrations of $\mathrm{Cu}, \mathrm{Cr}, \mathrm{Zn}, \mathrm{Cd}, \mathrm{Pb}, \mathrm{As}$, and $\mathrm{Ni}$ were above minimum safe concentration limits (interim sediment quality guideline - ISQG, CCME, 2002; threshold effect level TEL, USEPA, 2002), indicating possible harmful effects on aquatic organisms, according to Canadian and American Standards (CCME, 2002; USEPA, 2002). Copper (Cu) was above minimum safe concentration limit (ISQG: $35.7 \mathrm{mg} / \mathrm{kg}$ ) at all six sites of sector I. Chromium (Cr) was above minimum safe concentration limit (ISQG: $37.3 \mathrm{mg} / \mathrm{kg}$ ) at $56 \%$ of all sampling sites. Zinc (Zn) was above minimum safe concentration limit (ISQG: $123 \mathrm{mg} / \mathrm{kg}$ ) only at site 5 (sector I), while cadmium (Cd) was above minimum safe concentration limit of ISQG: 0.6 $\mathrm{mg} / \mathrm{kg}$ at $17 \%$ of sites, and at $17 \%$ of sites had minimum safe concentration limit. Lead $(\mathrm{Pb})$ was above minimum safe concentration limit (ISQG: $35.0 \mathrm{mg} / \mathrm{kg}$ ) at $50 \%$ of all sampling sites. Arsenic (As) and nickel (Ni) were above safe concentrations limits at all 18 sites of Lake Srebrno (ISQG (As): 5.9 mg/kg; CCME, 2002 and TEL (Ni): 18 mg/kg; USEPA, 2002). According to Canadian and American Standards (CCME, 2002; USEPA, 2002) arsenic and nickel were above minimum safe concentration limits indicating probable harmful effects on aquatic organisms (PEL - Probable Effect Level, CCME, 2002; USEPA, 2002). Arsenic was above PEL: $17 \mathrm{mg} / \mathrm{kg}$ at $17 \%$ of sites, and nickel was above PEL: $36 \mathrm{mg} / \mathrm{kg}$ at $94 \%$ of sites.

Pesticides (in Table 2 shown cumulative; $\mathrm{HCH}-\alpha, \beta, \delta$; lindane, heptachlor, heptachlor epoxide, alachlor, aldrin, dieldrin, endrin, DDT-DDE, DDD, hexachlorbenzol, atrazin, simazin and propazin) were not detected in the lake $(<10.0 \mu \mathrm{g} / \mathrm{kg})$ and neither was the presence of phenols recorded $(<10.0 \mu \mathrm{g} / \mathrm{kg})$. Polycyclic aromatic hydrocarbons (PAHs) were present at all sampling sites with concentrations between $36.4 \mu \mathrm{g} / \mathrm{kg}$ (site 12) and $524.8 \mu \mathrm{g} /$ $\mathrm{kg}$ (site 17). The following PAHs were above safe concentration limits, according to Canadian Standards (CCME, 2002) (Table 2 - shown cumulative): benzo (a) pyrene - in sectors I, II and IV, pyrene, anthracene and chrysene at site 11 (sector II) and naphthalene in sector IV. Polychlorinated biphenyls (PCBs) were under the detection limit $(<10.0 \mu \mathrm{g} / \mathrm{kg})$ at almost all investigated sites. Exceptionally, they were recorded at only two sampling sites of Sector I: $95.1 \mu \mathrm{g} / \mathrm{kg}$ (site 3) and $500.7 \mu \mathrm{g} / \mathrm{kg}$ (site 2), which indicates limited local pollution. At these two sites according to Canadian Standards (CCME, 2002) total PCBs was above minimum safe concentration limits (ISQG: $34.1 \mu \mathrm{g} / \mathrm{kg})$, and above PEL $(277 \mu \mathrm{g} / \mathrm{kg}$ ) indicating thus possible and probable harmful effects on aquatic organisms, respectively.

\section{Structure and diversity of the bottom fauna}

Qualitative bottom fauna composition of Lake Srebrno is presented in Table 3 as a list of all detected taxa, with their occurrence frequencies $(\mathrm{F}=0-1)$ at sampling sites.

The meio- and macrozoobenthic community of Lake Srebrno was composed of representatives of nine faunistic groups totalling 20 taxa: Chironomidae (9 taxa), Oligochaeta (4 taxa), Trichoptera (1 taxon), Hirudinea (1 taxon), Chaoboridae (1 taxon), while representatives of Nematoda, Diptera - Ceratopogonidae, Hydracarina and Bryozoa were not determined to the lower taxonomic levels.

The dominant group in the benthocenosis of Lake Srebrno was the Chironomidae group with the highest occurrence frequency $(\mathrm{F}=1)$ and with the greatest diversity $(9$ taxa). Subdominant group in the benthic community of the lake was Nematoda $(\mathrm{F}=0.89)$.

The lowest diversity of the bottom fauna community was at site 13 (one group only), 


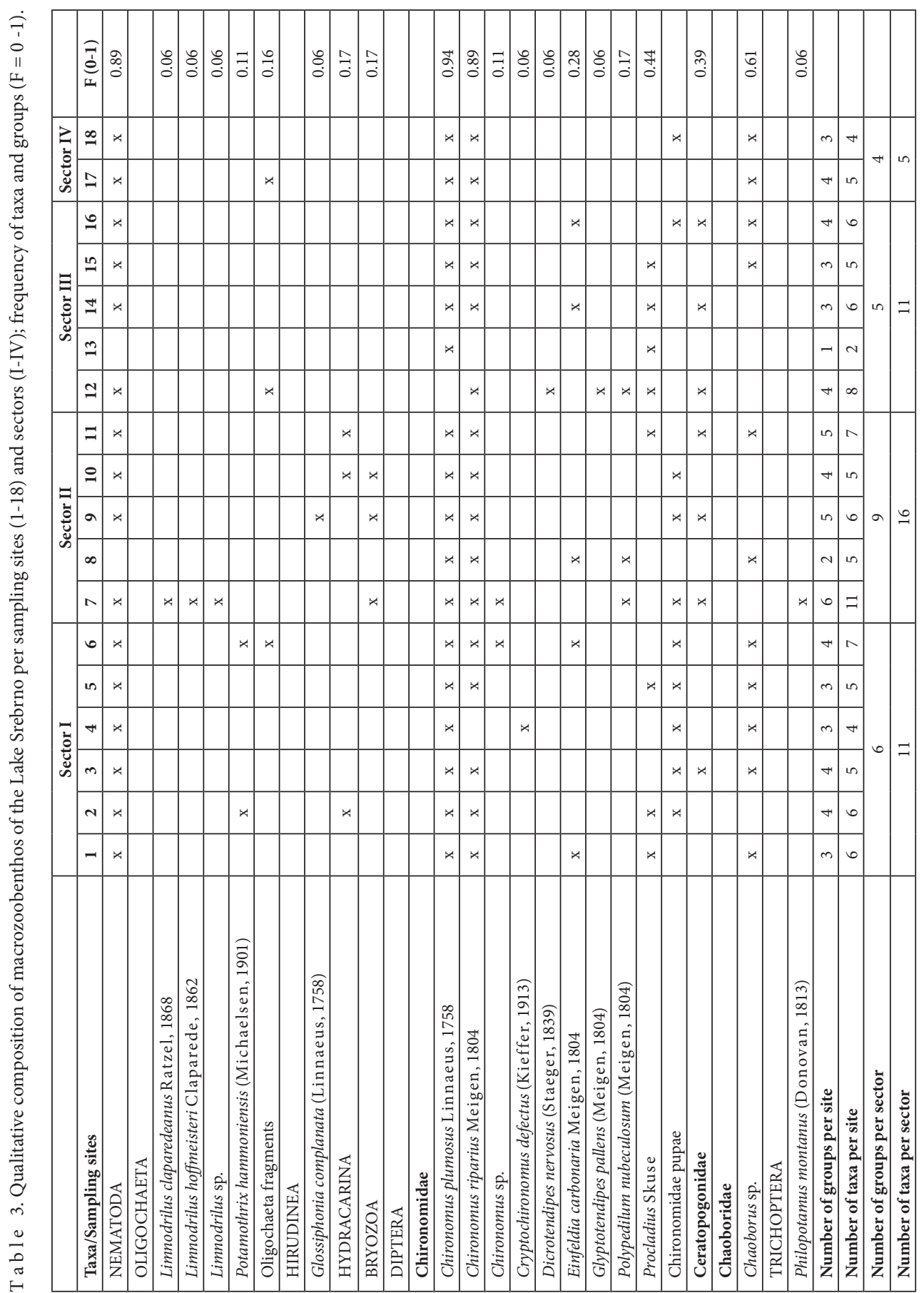


while the highest number of faunistic groups was at site 7 (six groups). Minimal number of taxa (2) was found at sampling site 13, and maximal number of taxa (11) was recorded at site 7. Sector IV had the lowest diversity of the bottom fauna community (4 group and 5 taxa), while the highest diversity was in sector II (9 groups and 16 taxa).

The most frequent and the most abundant species were Chironomus plumosus Lin naeus $(\mathrm{F}=0.94)$ and Chironomus riparius Meigen $(\mathrm{F}=0.89)$, whereas the lowest occurrence frequency $\mathrm{F}=0.06$ was recorded for: Limnodrilus hoffmeisteri Clap a rede, L. claparedeanus Ratzel, Limnodrilus sp., Cryptochironomus defectus (Kieffer), Dicrotendipes nervosus (Staeger), Gliptotendipes pallens (Meigen), Glossiphonia complanata (Linnaeus), and Philopotamus montanus (Donovan).

Correspondence analyses (CA) for macrozoobenthos of Lake Srebrno revealed statistically significant separation of sites and species in the space of the first two correspondence axes that accounted for $38.63 \%$ of the total variance. As shown in Fig. 2, the first correspondence axis (DIM 1) tended to separate site 7 (positive score on DIM 1).

This is in correlation with the presence of species inhabiting only this site (Limnodrilus

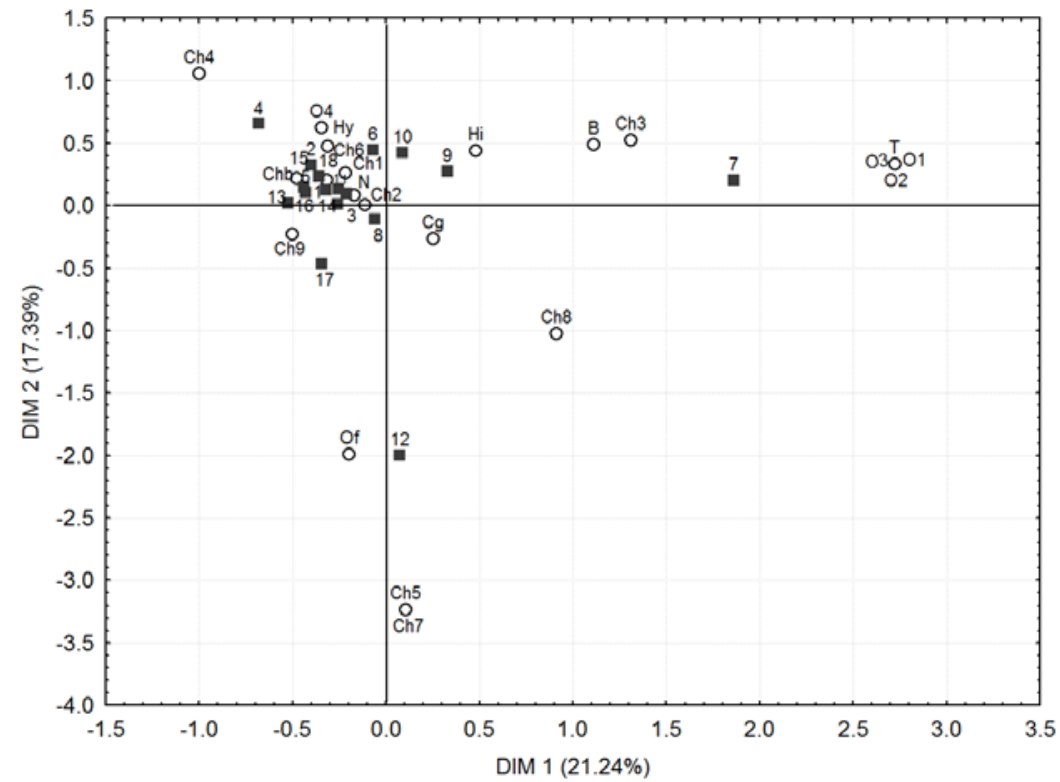

Fig. 2. Plot of macrozoobenthic species (circles) and sampling sites (squares) of the Lake Srebrno in the space of the first and the second correspondence axes (DIM). For abbreviations see Appendix.

sp., L. claparedeanus, L. hoffmeisteri and Philopotamus montanus). On the other hand, the second correspondence axis (DIM 2) tended to separate site 12 (negative score on DIM 2) and this is in correlation with the presence of two chironomid species (Dicrotendipes nervosus and Glyptotendipes pallens) which were recorded only at this sampling site. Sites 7 and 12 are characterized with the highest diversity (11 and 8 taxa, respectively). Whereas, $64 \%$ of all recorded species at site 7 had occurrence frequencies less than 0.20 in total macrozoobenthic community; while at site $12-50 \%$ of all had occurrence frequencies less than 0.20 . 
On the other hand, CA for macrozoobenthos of Lake Srebrno sectors revealed statistically significant separation of sectors and species in the space of the first two correspondence axes that accounted for $80.21 \%$ of the total variance. As shown in Fig. 3, the first correspondence axis (DIM 1) tended to separate Sector II (positive score on DIM 1).

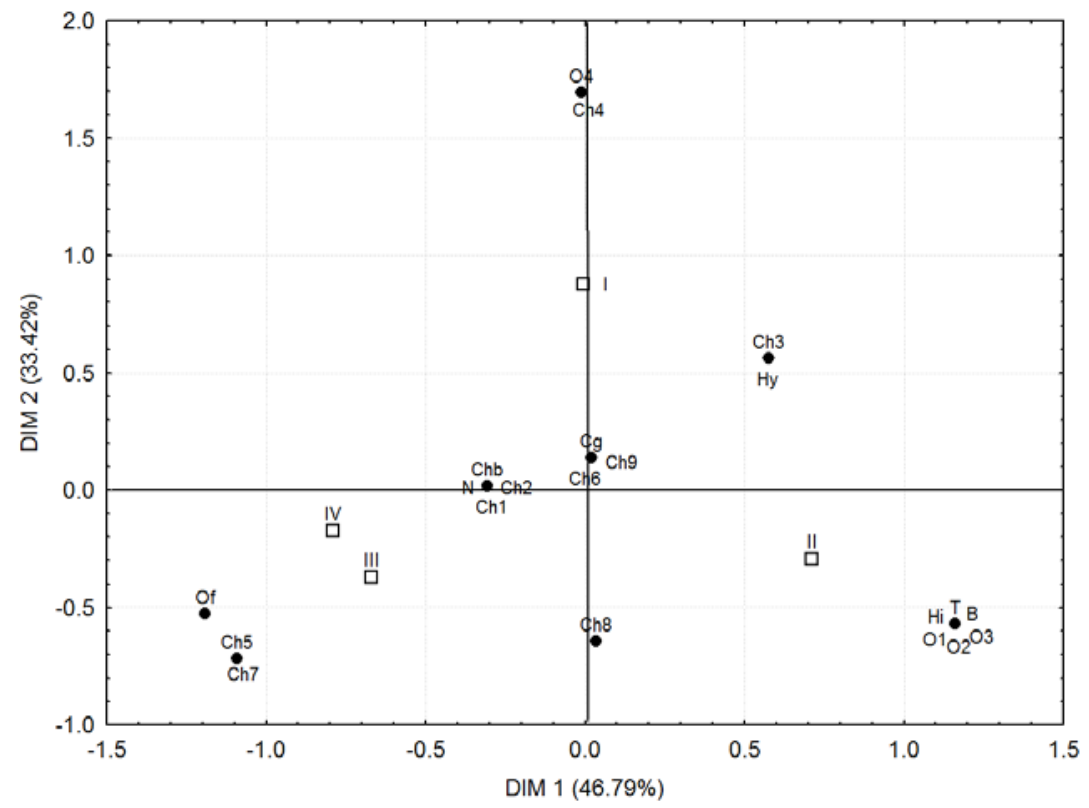

Fig. 3. Plot of macrozoobenthic species (circles) and sectors (squares) of the Lake Srebrno in the space of the first and the second correspondence axes (DIM). For abbreviations see Appendix.

This is in correlation with the presence of species that are found only in this sector (Limnodrilus sp, L. claparedeanus, L. hoffmeisteri, Glossiphonia complanata, Philopotamus montanus and Bryozoa). The second correspondence axis (DIM 2) tended to separate Sector I (positive score on DIM 2) and this is in correlation with the presence of Potamothrix hammoniensis and Cryptochironomus defectus, species which inhabiting only Sector I; while other recorded species in Sector I were very frequent species in the whole lake.

Quantitative bottom fauna composition of the Lake Srebrno and percentage participation in total density of bottom fauna is shown in Table 4.

The density of macrozoobenthos communities of Lake Srebrno varied between 481 ind. $\mathrm{m}^{-2}$ (site 13 , sector III) and 8,510 ind. $\mathrm{m}^{-2}$ (site 7 , sector II). However, at more than $40 \%$ of sites the density of macrozoobenthos communities ranged from 1,500 and 2,000 ind. $\mathrm{m}^{-2}$.

The Chironomidae group was dominant in all four sectors of Lake Srebrno. Their percentage participation in total macrozoobenthos abundance of the lake was between 53.7\% (site 8 ) and $100 \%$ (site 13). Chironomus plumosus was the dominant species in bottom fauna (12.9-84.6\% participation in lake), while Ch. riparius was subdominant species (7.7-38.7\% participation). 


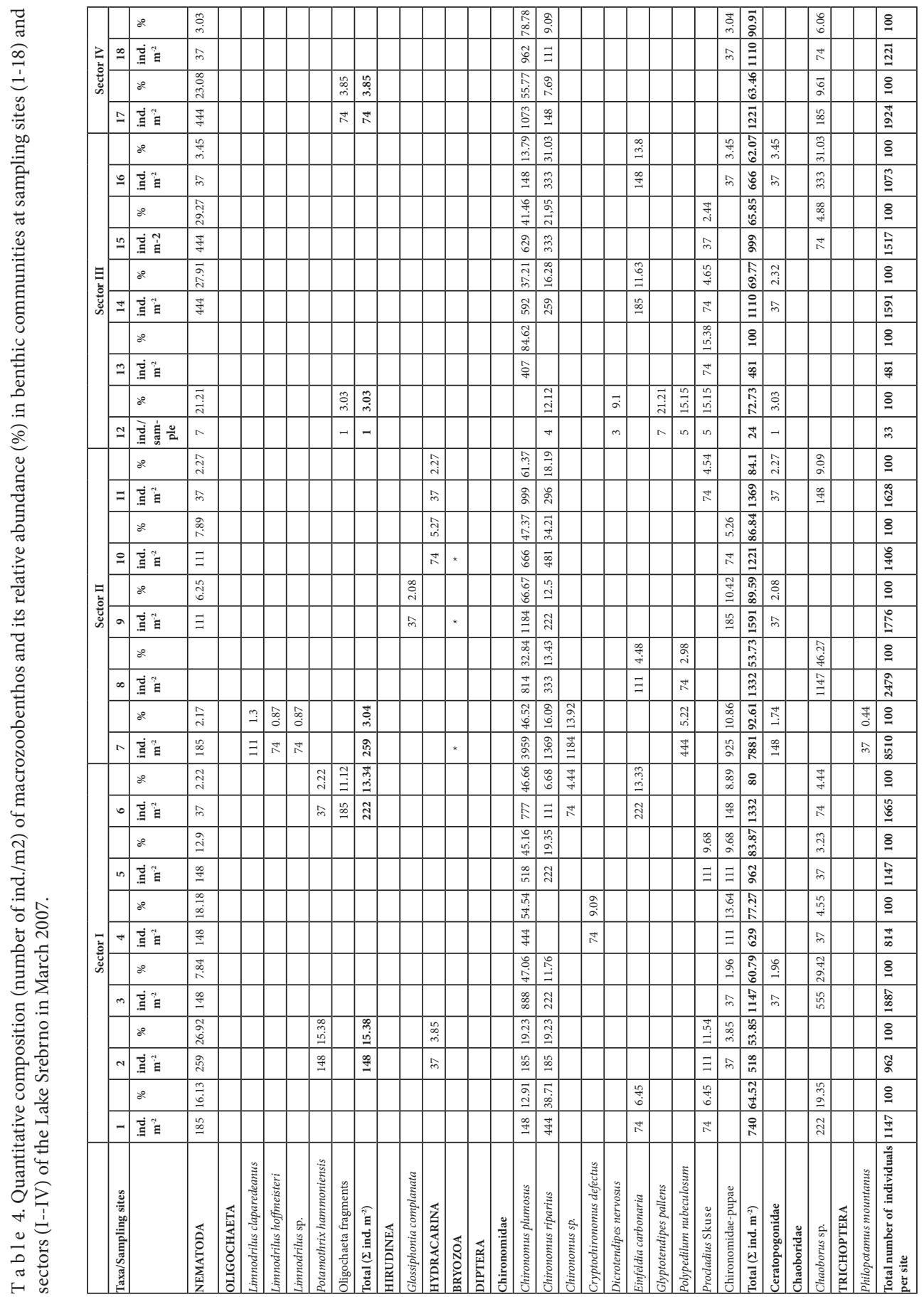


Another representative from Diptera group - species of genus Chaoborus was recorded in all four sectors of the lake, showed relatively high percentage participation in total macrozoobenthos abundance. This species was recorded at $61 \%$ of sites, and represents dominant species of bottom fauna at site 8 (II) $-46.3 \%$ (Table 4 ).

Occurrence frequency of Nematodes as subdominant zoobenthic group was 0.89 , even though the percentage participation of this group in the total macrozoobenthos abundance was not large, being from $2.2 \%$ (site 7 ) to $29.3 \%$ (site 15 ).

Representatives of Oligochaeta group were recorded in all sectors of the lake, at five sampling sites in all, whereas only fragments were recorded at sites 12 and 17, and their determination to the species level was not possible. Their participation in the total macrozoobenthos abundance was low (Table 4), relative abundance being between 3.0\% (site 12) and 15.4\% (site 2).

Quotients of Similarity (QS), according to Sørensen (1948), for benthic communities of Lake Srebrno are shown in Tables $5 \mathrm{a}$ and $5 \mathrm{~b}$.

T a b le 5a. Values of Sørensen's Quotient of Similarity (QS) (Sørensen, 1948) based on bottom fauna communities per sampling sites (1-18) in the Lake Srebrno in March 2007.

\begin{tabular}{|c|c|c|c|c|c|c|c|c|c|c|c|c|c|c|c|c|c|}
\hline Sampling site & & & & & & & & & & & & & & & & & \\
\hline & 1 & & & & & & & & & & & & & & & & \\
\hline 1 & & 2 & & & & & & & & & & & & & & & \\
\hline 2 & 66.77 & & 3 & & & & & & & & & & & & & & \\
\hline 3 & 72.73 & 54.55 & & 4 & & & & & & & & & & & & & \\
\hline 4 & 60.00 & 40.00 & 66.67 & & 5 & & & & & & & & & & & & \\
\hline 5 & 90.91 & 54.55 & 80.00 & 66.67 & & 6 & & & & & & & & & & & \\
\hline 6 & 76.92 & 61.54 & 66.67 & 54.55 & 66.67 & & 7 & & & & & & & & & & \\
\hline 7 & 35.29 & 35.29 & 50.00 & 26.67 & 37.50 & 44.44 & & 8 & & & & & & & & & \\
\hline 8 & 72.73 & 36.36 & 60.00 & 44.44 & 60.00 & 66.67 & 37.50 & & 9 & & & & & & & & \\
\hline 9 & 50.00 & 50.00 & 72.73 & 40.00 & 54.55 & 46.15 & 58.82 & 36.36 & & 10 & & & & & & & \\
\hline 10 & 54.55 & 72.73 & 60.00 & 44.44 & 60.00 & 50.00 & 50.00 & 40.00 & 72.73 & & 11 & & & & & & \\
\hline 11 & 76.92 & 76.92 & 83.33 & 54.55 & 83.33 & 57.14 & 44.44 & 50.00 & 61.54 & 66.67 & & 12 & & & & & \\
\hline 12 & 42.86 & 42.86 & 46.15 & 16.67 & 46.15 & 26.67 & 42.10 & 30.77 & 42.86 & 30.77 & 53.33 & & 13 & & & & \\
\hline 13 & 50.00 & 50.00 & 28.57 & 33.33 & 57.14 & 22.22 & 15.38 & 28.57 & 25.00 & 28.57 & 44.44 & 20.00 & & 14 & & & \\
\hline 14 & 83.33 & 66.67 & 72.73 & 40.00 & 72.73 & 61.54 & 47.06 & 54.55 & 66.67 & 54.55 & 76.92 & 57.14 & 50.00 & & 15 & & \\
\hline 15 & 90.91 & 72.73 & 80.00 & 66.67 & 100 & 66.67 & 37.50 & 60.00 & 54.55 & 60.00 & 83.33 & 46.15 & 57.14 & 72.73 & & 16 & \\
\hline 16 & 83.33 & 50.00 & 90.91 & 60.00 & 72.73 & 76.92 & 47.06 & 72.73 & 66.67 & 54.55 & 76.92 & 42.86 & 25.00 & 83.33 & 72.73 & & 17 \\
\hline 17 & 72.73 & 54.55 & 80.00 & 66.67 & 80.00 & 66.67 & 37.50 & 60.00 & 54.55 & 60.00 & 66.67 & 46.15 & 28.57 & 54.55 & 80.00 & 72.73 & \\
\hline 18 & 80.00 & 60.00 & 88.89 & 75.00 & 88.89 & 72.73 & 40.00 & 66.67 & 60.00 & 66.67 & 72.73 & 33.33 & 33.33 & 60.00 & 88.89 & 80.00 & 88.89 \\
\hline
\end{tabular}

T a b le 5b. Values of Sørensen's Quotient of Similarity (QS) (Sørensen, 1948) based on bottom fauna communities per sectors (I-IV) in the Lake Srebrno in March 2007.

\begin{tabular}{|l|c|c|c|c|c|c|c|c|c|c|c|c|}
\hline Sectors & I & II & I & III & I & IV & II & III & II & IV & III & IV \\
\hline Number of species & 11 & 16 & 11 & 11 & 11 & 5 & 16 & 11 & 16 & 5 & 11 & 5 \\
\hline Number of species in common & \multicolumn{2}{|c|}{9} & \multicolumn{2}{|c|}{7} & \multicolumn{2}{|c|}{4} & 7 & & 4 & 5 \\
\hline QS (\%) & \multicolumn{2}{|c|}{66.67} & \multicolumn{2}{|c|}{63.64} & \multicolumn{2}{|c|}{50} & 51.85 & \multicolumn{2}{|c|}{38.09} & 62.5 \\
\hline
\end{tabular}


The minimum degree of similarity - $15.4 \%$ was detected between site 7 and 13, and maximum degree of similarity - 100\% was detected between site 5 and 15 . Relatively great number of sites - $21 \%$ had faunistic similarity between $51 \%$ and $60 \%$. Another $20 \%$; $18 \%$; $13 \%$; and $10 \%$ of sites showed a similarity between $71-80 \% ; 41-50 \% ; 61-70 \%$ and $31-40 \%$, respectively. A smaller number of sites $-8 \%$ and $7 \%$ showed a similarity between $81 \%$ and $90 \%$ and $21-30 \%$, respectively. The lowest faunistic similarity (11-20\%) was detected for just $2 \%$ of sites, and the highest similarity (91-100\%) was detected for $0.6 \%$ of sites. Similarity less than $10 \%$ was not detected.

Table 6 summarizes the results of saprobiological bottom fauna analysis, based on Saprobic index (S) calculations (Pantle, Buck 1955), and classification of saprobity levels, according to Moog (2002) with the water quality classes, as well as relationship of saprobity and trophy after Martinovic-Vitanovic et al. (2008).

$\mathrm{T}$ a b l e 6. Water quality of the Lake Srebrno in Serbia based on saprobiological analysis of benthic communities and the correspond trophy level at the sampling sites (1-18) and sectors (I-IV) in March 2007.

\begin{tabular}{|c|c|c|c|c|c|c|c|c|c|c|c|c|c|c|c|c|c|c|}
\hline \multirow{2}{*}{$\begin{array}{l}\text { Sector } \\
\text { Sampling sites }\end{array}$} & \multicolumn{6}{|c|}{ I } & \multicolumn{5}{|c|}{ II } & \multicolumn{5}{|c|}{ III } & \multicolumn{2}{|c|}{ IV } \\
\hline & 1 & 2 & 3 & 4 & 5 & 6 & 7 & 8 & 9 & 10 & 11 & 12 & 13 & 14 & 15 & 16 & 17 & 18 \\
\hline Saprobity index $S$ & 3,54 & 3,27 & 3,56 & 3,21 & 3,56 & 3,41 & 3,24 & 3,36 & 3,43 & 3,55 & 3,56 & 2,81 & 3,60 & 3,56 & 3,56 & 3,54 & 3,56 & 3,58 \\
\hline Saprobity level & $p-\alpha$ & $\alpha$ & $p-\alpha$ & $\alpha$ & $p-\alpha$ & $\alpha$ & $a$ & $\alpha$ & $\alpha$ & $\mathrm{p}-\alpha$ & $p-\alpha$ & $a$ & $p-\alpha$ & $p-\alpha$ & $p-\alpha$ & $p-\alpha$ & $\mathrm{p}-\alpha$ & $\mathrm{p}-\alpha$ \\
\hline Classis & IV-III & III & IV-III & III & IV-III & III & III & III & III & IV-III & IV-III & III & IV-III & IV-III & IV-III & IV-III & IV-III & IV-III \\
\hline Trophy level & hyper- & eu- & hyper- & eu- & hyper- & eu- & eu- & eu- & eu- & hyper- & hyper- & eu- & hyper- & hyper- & hyper- & hyper- & hyper- & hyper- \\
\hline
\end{tabular}

Notes: alphamesosaprobity- a vs. eutrophy- eu-; poly-alphamesosaprobity- p-a vs. hypertrophy- hyper-.

In Lake Srebrno out of 18 sites in all, $61.1 \%$ of sites were in IV-III class limits corresponding to hypertrophy, while another $38.9 \%$ of sites were in the range of III water quality class which corresponds to eutrophy.

\section{Discussion}

Morphometric and ecological classification of Lake Srebrno, consider ecosystem of the lake in transition to plain marsh ecosystem - pseudo-lake, i.e. shallow lake only with littoral zone, without profundal. It is the case with some other aquatic ecosystems - in Serbia: Obedska Bara marsh, Sava Lake, lakes Palic and Ludos, and Zobnatica reservoir; and - in Croatia Lake Jarun. The difference between them refers to their origin; Obedska Bara marsh is a natural system originating by the Sava River flooding, lakes Ludos and Palic are primarily swamps of Aeolian origin before the water regulation system in the area, while Lake Srebrno, Sava Lake, Zobnatica reservoir and Lake Jarun are artificial lakes (Martinovic-Vitanovic, 1996; Martinovic-Vitanovic, Kalafatic, 1999; Martinovic-Vitanovic et al., 2006, 2008, 2009/2010; Selesi, 1979; Kerovec et al., 1989).

Trophic status of these aquatic ecosystems based on Chl-a concentration values and macrozoobenthos as an indicator of the saprobity and trophy was generally defined as eutrophic (Jankovic et al., 1983, 1988; Kerovec et al, 1989; Martinovic-Vitanovic, 1996; Martinovic-Vitanovic, Kalafatic, 1999; and Selesi, 1979). Water transparencies and chlorophyll $a$ concentrations, according to the classification schemes for lake water trophy (OECD, 1982) showed 
that Lake Srebrno in March 2007 had a eutrophic status (the standard - minimal water transparency for eutrophic water $-1.5-0.7 \mathrm{~m})$. Chlorophyll- $a$ concentration per sites were in wide interval, ranged from $18.50 \mu \mathrm{g} / \mathrm{l}$ (site 13) to $54.76 \mu \mathrm{g} / \mathrm{l}$ (site 18). Concentrations of Chl- $a$ at $61 \%$ of sites were above $25 \mu \mathrm{g} / \mathrm{l}$ (the standard - maximal Chl- $a$ concentration for eutrophic waters of $25-75 \mu \mathrm{g} / \mathrm{l}$ ), while at $33 \%$ of sites Chl- $a$ concentrations "reduced" mean value to the upper limit of mesotrophy and in transition to eutrophy (the standard - maximal Chl- $a$ concentration for mesotrophic waters of $8-25 \mu \mathrm{g} / \mathrm{l})$.

Agricultural activities around the lake, as a source of direct pollution, as well as input of pollutants from Kisiljevo stream and Ostrovo canal, and municipal wastewater from villages and weekend cottages, resulted in great input of nutrients. Thus, by lake autochthonous and allochthonous progressive enrichment (Jankovic et al., 1983; 1988) in favourable climate conditions, and with morphometric characteristics of shallow water bodies, established rapid formation of rich substrate - silt. Therefore, silt is present at all sites of Lake Srebrno, and it smells like $\mathrm{H}_{2} \mathrm{~S}$.

Based on Martinovic-Vitanovic and Kalafatic habitat classification (Martinovic-Vitanovic, Kalafatic, 1995; Lakusic et al., 2005), in Lake Srebrno, in the uniform habitats, benthic communities are predominant on the fine-grained bottom (code 2.1.2. - particles between 0.01 and $1 \mathrm{~mm}$ ). That includes $67 \%$ of sites (12 sites), out of which seven bottom facies belong to type on which benthic communities of silt bottom develop (code 2.1.2.2. - particles between 0.01 and $0.1 \mathrm{~mm}$ ), another five bottom facies belong to type on which benthic communities of fine-grained sand bottom develop (particles between 0.1 and $1 \mathrm{~mm}-$ code 2.1.2.3.). Bottom facies belong to bottom type on which benthic communities of fine-grained bottom develop (code 2.1.1. - particles $<$ than $0.01 \mathrm{~mm}$ ) was present at another $33 \%$ of sites (six sites), which includes bottom type on which benthic communities of fine-fractioned silt bottom develop (code 2.1.1.4.). Exception is site 6 (sector I) where prevailing granulation particles are those that belong to facies type on which benthic communities of hard bottom develop (0.1-1 cm - coded as: 2.1.3.1.). To partially heterogenic habitat contributes presence of sand and pebble, recorded at $28 \%$ of sites, whereas mixed type of substrate is recorded in sector II (sites: 7, 9 and 11), while its presence in sectors I and III is limited at one site only (6 and 12 , respectively).

In some shallow lakes, like Lake Balaton, light is primarily determined by sediment resuspension induced by wind, and that decisively determines the underwater light climate at the present trophic state of the lake (Istvanovics et al., 2008). During 2007 investigation period, in Lake Srebrno low water transparency was recorded (Secchi disc depth $<1.5 \mathrm{~m}$ ). Due to the facts that the silt was predominant in substrate, that the lake is in north-eastern Serbian district where strong wind "Kosava" dominates, and that it is relatively shallow lake (maximal depth was $7 \mathrm{~m}$ ), according to OECD standard (1982), Lake Srebrno had eutrophic status. In addition to this, in previous, 2006 investigations was detected high primary and secondary plankton production, as a result of extreme abundant phyto- and zooplankton populations. That affected on low water transparency, being in the range of eutrophy, and mostly in hypertrophy (OECD, 1982; Martinovic-Vitanovic, Kalafatic, 2006).

Regarding to heavy metal concentrations in the lake sediments, lake Srebrno can be compared with lake Balaton in which anthropogenic activities in and around the lake induced higher concentrations of $\mathrm{Cr}, \mathrm{Mn}, \mathrm{Co}$ and $\mathrm{Ni}$ in the lake sediments as well (Nguyen et al., 
2005). Sector I of Lake Srebrno was the most loaded with heavy metals (As, Cu, Zn, Cr, $\mathrm{Cd}, \mathrm{Ni}$, and $\mathrm{Pb}$ ) as well with polychlorinated biphenyls (PCBs). Limited local pollution with PCBs is probably a result of removing a transformer station from tourist centre (Sector I, sites 2 and 3.). A possible reason for the presence and enhanced concentrations of some polycyclic aromatic hydrocarbons (PAHs) is accelerated urbanization in the investigated area. PAHs usually come from wastewater and arise as intermediate degradation products of some organic (phyto) materials; also, petroleum being a source (Martinovic-Vitanovic, 1996). The enhanced concentrations of arsenic and nickel in Lake Srebrno are likely to have harmful effects on the benthic community, as Laliberte, Tremblay (2002) find it stands for sediments from four lakes in Northern Quebec. According to Bobori (1996), all metal concentrations in Lake Koronia sediments were found much higher than those in water, confirming the ability of the lake sediment to act as a pool for heavy metals. However, total number of groups and taxa of bottom fauna per Lake Srebrno sectors did not significantly varied in relation to presence/ absence of pollutants. According to Dévai (1990), neither the quantitative nor the qualitative occurrence of heavy metals and chlorinated hydrocarbons, singly or together, were related to the spatial pattern of the chironomid communities in Lake Balaton. The fact that no Lake Srebrno sediment samples contained measurable quantities of mercury, cyanides, phenols and pesticides illustrate favourable conditions for lake biota.

Benthocenoses of Lake Srebrno showed low diversity and had a composition and a structure similar to those of other artificial and natural ("flood plain" type) lowland lakes formed in large river valleys in Serbia such as Sava Lake, Obedska Bara marsh, some Pannonian lakes, and the others in Danube and Sava river basins (Jankovic, 1967, 1974, 1982; Jakovcev, 1989; Martinovic-Vitanovic, 1996; Martinovic-Vitanovic, Kalafatic, 1999; Selesi, 1979; Martinovic-Vitanovic et al., 2006, 2008, 2009/2010). Due to the absence of vegetation at the time of sampling, especially submerged vegetation, in addition to substrate type previously discussed, the diversity of Lake Srebrno bottom fauna was not high, and generally uniform, as characteristic for mentioned standing water in Serbia. On the other hand, hydrological connectivity between the main river stream and the associated floodplain water bodies is the key factor influencing the biodiversity and distribution of species in floodplain water bodies (Reckendorfer et al., 2006).

Out of 20 taxa in all, 45\% of taxa are from family Chironomidae (Diptera), and 20\% of taxa are from family Tubificidae (Oligochaeta). Hirudinea, Trichoptera and Chaoboridae (Diptera) were presented by one taxon each, whereas Nematoda, Hydracarina, Bryozoa and Ceratopogonidae (Diptera) were not determined to the lower taxonomic levels. Unusually for this type of aquatic ecosystem, at the time of sampling there was a complete absence of the representatives of Mollusca group, an important component of the bottom fauna of lakes Sava and Jarun (Jakovcev, 1989; Kerovec et al, 1989). Obolewski et al. (2009) found that temporarily harsh environment of shallow, isolated lenthic lakes with low oxygen content and low transparency could completely exclude bivalves, while long-term oxygen deficits in lenthic reservoirs can cause the withdrawal of gastropods from bottom onto the macrophytes. On the other hand, gastropods preferring flowing water, and they are sensitive to the low levels of water, high temperature and low transparency. Therefore, this intolerance is probable reason of molluscs' absence in Lake Srebrno.

Dominant group of lake Srebrno benthic fauna - Chironomidae was the most diversified 
(five taxa) at sampling site 12 (sector III). In relation to other faunistic groups, the great diversity of this insect group was due to the presence of nine taxa belonging to two families: Chironominae (eight taxa) and Tanypodinae (one taxon). Species of the genus Chironomus were present at all sampling sites. They represent bioindicators for the presence of organic pollutants, and were used for the assessment of the eutrophication of the lake. Chironomus plumosus occurs in lakes with high eutrophic levels (Resh, Rosenberg, 1984; Dudley, Feltmate, 1992; Kagalou et al., 2006). Chironomus riparius has a widespread distribution in the northern hemisphere, mostly in the temperate zone, and is a common resident of polluted waters (Lindegaard, 1995). Larvae of those species are tolerant to a wide range of variations of $\mathrm{pH}$, oxygen concentration and diameter of sediment particles (Ristola, 2002). Because of the dominance of species of the genus Chironomus in the bottom fauna, Lake Srebrno could be classified as a eutrophic lake of the Chironomus type (Lindegaard, 1995; Martinovic-Vitanovic, 1996). Another frequent chironomid - Procladius Skuse was recorded at $44 \%$ of sites. According to Specziar, Biro (1998) Procladius seems to inhabit lakes and reservoirs in a wide range of trophic state from oligotrophy to hypertrophy. Generally, Chironomus - Procladius community indicate moderate euthrophy (Dévai, 1990; Specziar, Biro, 1998). Nevertheless, chironomids play an important role in preserving sediment quality and in natural prevention of the eutrophication, because chironomids remove organic carbon, nitrogen and phosphorous from the lake (Dévai, 1990).

Apart from family Chironomidae, Diptera group was presented in Lake Srebrno with another two families: Chaoboridae $(\mathrm{F}=0.61)$ and Ceratopogonidae $(\mathrm{F}=0.39)$. Presence of the genus Chaoborus (Chaoboridae), which is usual in stagnant water with unfavourable oxygen conditions at the lowest water layers (Jankovic, 1974; Martinovic-Vitanovic, 1996; Martinovic-Vitanovic, Kalafatic, 1999; Kagalou et al., 2006), indicates high levels of trophy and saprobity. Out of 11 sites in all where species of genus Chaoborus was recorded in Lake Srebrno, eight sites had hypertrophic, and three sites had eutrophic status.

Representatives of Oligochaeta in Lake Srebrno were widespread pelophylic species of the Tubificidae family: Limnodrilus hoffmeisteri, L. claparedeanus and Potamothrix hammoniensis. In addition to these three tubificid species, another species of genus Limnodrilus was recorded at site 7, but its determination to the species level was not possible. According to Verdonschot (1999), Limnodrilus hoffmeisteri prefers fine sand with a medium to high organic matter content and coarse sand with medium organic matter content and avoids all other substrates. In March 2007, L. hoffmeisteri was recorded only at site 7 (II) which was characterized by fine sand and coarse detritus. Potamothrix hammoniensis well developed in high trophic conditions (Risnoveanu, Vadineanu, 2002, 2003). In lake Srebrno this species was recorded at sites (2 and 6) characterized by eutrophic conditions.

The Oligochaeta participation in the total macrozoobenthos abundance was low, relative abundance being between $3.0 \%$ (site 12) and 15.4\% (site 2). As well as low presence, concerning the fact that all annotated taxa originated from only one family, Oligochaeta of Lake Srebrno had shown low diversity and low abundance. The identified species of Oligochaeta indicate organic pollution, as they are known to be tolerant to oxygen deficiency in the substrate and in the contact layer. The low abundance of Oligochaeta and their proportionally small contribution to total macrozoobenthic abundance could be due to seasonal dynamics. Samples were collected in March 2007, and Oligochaeta have a tendency to increase their abundance from spring to summer, with maximum in June (Jankovic, 1974). Many tubificid species in 
temperate regions have one reproductive period a year, often from May to July (Brinkhurst, 1964), and due to the fact that our investigations were conducted in March, low abundance of Oligochaeta was expected. According to Verdonschot (1999), temporal and spatial distribution of oligochaetes cannot be separated, and season-abundance relationship is more significant than substrate-abundance relationship, because of that low abundance is probably associated with investigated period.

The uniform bottom fauna community of Lake Srebrno was probably due to substrate homogeneity. The exception is Sector II, which differs from other three sectors of the lake due to the highest diversity (9 groups and 16 taxa). Namely, only in this sector were recorded representatives of Hirudinea, Bryozoa and Trichoptera. This sector is affected by the Kiseljevo stream flowing through village brings higher organic pollution. Site 7 (sector II) - the mouth of the Kisiljevo brook, was different from other sites by composition, diversity and abundance of bottom fauna, having the greatest taxa diversity -6 groups and 11 taxa, and maximal abundance $-8,510$ ind. $\mathrm{m}^{-2}$. In addition, this site was also the shallowest one $(2.5 \mathrm{~m})$, and coarse detritus and erosion materials were being imported by the Kisiljevo brook, which implies back filling.

According to Sørensen Quotient of Similarity (QS) medium to high degrees of similarity between benthocenoses were found amongst all 18 sampling sites. The majority of sites $-56 \%$ have medium similarity values (21-60\%). The high QS values between 61 and $100 \%$ have $42 \%$ of sites, while similarity between 11 and $20 \%$ was detected for just $2 \%$ of sites. In addition to this, medium to high degrees of similarity of benthocenoses were recorded across the four Sectors, varying from 38\% between Sectors I and IV and 67\% between Sectors I and II. The high faunistic similarity recorded between sites was the result of abundant taxa typical for soft sediments, which were detected at every sampling site of the lake. Faunistic dissimilarities between Lake Srebrno sites resulted from the presence of representatives of Oligochaeta, Hydracarina, and Bryozoa groups that were detected at few sites, and the sporadic presence of species of Hirudinea, Trichoptera - Philopotamus montanus (Donovan), and Chironomidae -Polypedilum nubeculosum (Meigen), Cryptochironomus defectus (Ki effer), Dicrotendipes nervosus (Sta eger) and Glyptotendipes pallens (Meigen).

Qualitative and quantitative analyses of benthocenoses, and especially the Chironomidae group, were used to assess the trophic levels in aquatic ecosystems (Jonasson, 1972; Kerovec et al., 1989; Dévai, 1990; Johnson et al., 1993; Armitage et al., 1995; Lindegaard, 1995; Kagalou et al., 2006). Representatives of the groups Chaoboridae, Chironomidae and Oligochaeta that were recorded in Lake Srebrno are faunistic elements of eutrophic and polluted waters (Jankovic, 1974).

The results presented in this study, based on analyses of benthocenosis composition and structure, as well as saprobiological analysis, indicate that Lake Srebrno is a eutrophic in transition to hyper-eutrophic ecosystem. This classification was confirmed with physical and chemical analyses of the water and silt, as well as concentrations of Chl- $a$. Eutrophication is characteristic for this type of water basin (shallow, lowland water bodies), but in this case it is increased by anthropogenic factors with input of pollutants from village and weekend-cottages wastewaters, swilling out of agricultural areas, as well as input of water from Kisiljevo stream and Ostrovo canal. Recorded macrozoobenthic community at sampling sites of Lake Srebrno was uniform. This was confirmed by medium to high Quotients of Similarity between benthocoenoses, which was found amongst the four sectors of Lake Srebrno, with the highest simi- 
larity between Sectors I and II (66.7\%). The soft sediments that predominate resulted in the presence and abundance of taxa usual for this habitat type. The presence of species from the Chaoboridae, Chironomidae and Oligochaeta groups is typical for eutrophic and polluted waters with high trophy and saprobity levels. Thus, Lake Srebrno in March 2007 corresponds to alpha-mesosaprobic conditions - eutrophy, and being in the range from poly- to alpha-mesosaprobity - hypertrophy. As artificial aquatic ecosystem Lake Srebrno was among others studied for the characterization and assessment of the ecological status of standing waters as lay down in the EU-Water Framework Directive in order to support its implementation in Serbia, and was denoted as anthropogenically "substantially modified water body".

Translated by the authors English corrected by S. Quarrie

\section{Acknowledgements}

This study was supported by the Ministry of Science and Technological Development of the Republic of Serbia (Fundamental Investigations - Grant No. 146021) and the Serbian Ministry of Education, Science and Technological Development (Fundamental Investigations - Grant No. 176018) and was conducted in collaboration with the stuff of the Institute of Public Health, Belgrade. For improving the English we are thankful to Mrs. Danka Filipovic, Lector of English at Faculty of Biology, University of Belgrade and to Dr. Steve Quarrie, native English speaker, visiting professor at Newcastle University, U.K.

\section{References}

APHA-AWWA-WEF. (1995). Standard methods for the examination of water and wastewater. Washington DC, USA: American Public Health Association.

Armitage, P.D., Cranston P.S. \& Pinder L.C. (1995). The Chironomidae: the biology and ecology of nonbiting midges. London: Chapman and Hall.

Bobori, D. (1996). Bioaccumulation of heavy metals in the ecosystem of Lake Koronia (Macedonia, Greece). Bios (Macedonia, Greece), 4, 171-174.

Brinkhurst, R. O. (1964). Observations on the biology of lake-dwelling Tubificidae. Arch. Hydrobiol., 60, 385-418.

Brinkhurst R.O. \& Jamieson B.G.M. (1971). Aquatic Oligochaeta of the world. Edinburgh: Oliver \& Boyd.

CCME. (2002). Canadian Sediment Quality Guidelines for the Protection of Aquatic Life: Summary tables. Updated. Winnipeg: Canadian Council of Ministers of the Environment.

Chapman, D. (Ed.) (1997). Water quality assessment. A Guide to the use of biota, sediments and water in environmental monitoring. 2nd ed. London: Chapman \& Hall.

Croft, P.S. (1986). A key to the major groups of British freshwater invertebrates. Field Studies, 6, 531-579.

Dévai, Gy. (1990). Ecological background and importance of the change of chironomid fauna (Diptera: Chironomidae) in shallow Lake Balaton. Hydrobiologia, 191, 189-198. DOI:10.1007/BF00026052.

Dudley W.D. \& Feltmate B.W. (1992). Aquatic insects. Wallingford, Oxon: C.A.B. International.

Elliot J.M. \& Mann K.H. (1979). A key to the British freshwater leeches. Ambleside: Freshwater Biological Association.

Istvánovics, V., Honti, M., Kovács, A. \& Osztoics A. (2008). Distribution of submerged macrophytes along environmental gradients in large, shallow Lake Balaton (Hungary). Aquat. Bot., 88, 317-330. DOI:10.1016/j.aquabot.2007.12.008.

Jakovcev, D. (1989). The composition of macrobenthic fauna of Sava Lake at Belgrade (in Serbian). Biosistematika Belgrade, 15(1), 41-47.

Jankovic, M. (1967). A contribution of the knowledge of Chironomidae of Serbia (in Serbian). Bull. Mus. Hist. Nat., $22,172-179$.

Jankovic, M. (1974). A contribution of the knowledge of aquatic cenosis of Obedska Bara (in Serbian). Transactions Institute for Nature Conservation Serbia, 1(9), 1-22.

Jankovic, M. (1982). A contribution to the knowledge of benthic fauna in the marsh Obedska Bara (in Serbian). Nat. Sci. Repertory Matica Srpska Novi Sad, 62, 137-150.

Jankovic, M.M., Jankovic, J.M., Kalafatic V. \& Martinovic-Vitanovic V. (1983). Study of increasing biomass in Ada Ciganlija Lake and effects of its suppression. Study of riverbanks, rivers' littoral region and rivers of Belgrade - "Hidrozavod" (in Serbian). Belgrade: Project of the City Government of Belgrade. 
Jankovic, M.M., Jankovic, J.M., Kalafatic, V. \& Lazarevic M.M. (1988). An ecological aspect of the Sava Lake by Belgrade (Ada Ciganlija) in regard to its overgrowth, purification and sanitation (especially in relation to the role of macrophytes in eutrophication overgrowth of the lake ecosystem) II (in Serbian). Ekologija Belgrade, 23, 65-116.

Johnson, R.K., Wiederholm T. \& Rosenberg D.M. (1993). Freshwater biomonitoring using individual organisms, population and species assemblages of benthic macroinvertebrates. In D.M. Rosenberg \& V.H. Resh (Eds.), Freshwater Biomonitoring and Benthic Macro-invertebrates (pp. 40-158). New York: Chapman and Hall.

Jonasson, P.M. (1972). Ecology and production of the profundal benthos in relation to phytoplankton in lake Esrom. Oikos, 14(Suppl), 1-148.

Kagalou, I., Economidis, G., Leonardos, I. \& Papaloukas C. (2006). Assessment of a Mediterranean shallow lentic ecosystem (Lake Pamvotis, Greece) using benthic community diversity: Response to environmental parameters. Limnologica, 36, 269-278. DOI:10.1016/j.limno.2006.08.002.

Kerovec, M., Tavcar, V. \& Mestrov M. (1989). Macrozoobenthos as an indicator of the level of the trophy and saprobity of Lake Jarun. Acta Hydrochim. Hydrobiol., 17, 1, 37-45. DOI:10.1002/aheh.19890170106.

Lakusic, D., Blazencic, J., Randjelovic, V., Butorac, B., Vukojicic, S., Zlatkovic, B., Jovanovic, S., Sinzar-Sekulic, J., Zukovec, D., Calic I. \& Pavicevic D. (2005). Habitats of Serbia - Manual with descriptions and basic data (in Serbian). In D. Lakušić (Ed.), Habitats of Serbia, Project results „Harmonization of national nomenclature in the classification of habitats with standards of International Community". Belgrade: Institute of Botany and Botanical Garden "Jevremovac", Faculty of Biology, University of Belgrade, Ministry of Science and Environmental Protection of the Republic of Serbia.

Laliberte, D., \& Tremblay, G. (2002). Metal, PCB, dioxin and furan concentration in fish and sediment from four lakes in Northern Quebec in 2001. Quebec: Ministere de l'Environnement. (Report no. QE/129)

Lellak, J. (1980). Pakomárovití — Chironomidae (in Czech). In R. Rozkošný (Ed.), Klíč vodních larev hmyzu (pp. 310— 392). Praha: Academia.

Lindegaard, C. (1995). Classification of water-bodies and pollution. In P.D. Armitage, P.S. Cranston \& L.C. Pinder (Eds.), The Chironomidae: the biology and ecology of nonbiting midges (pp. 385-404.). London: Chapman and Hall.

Martinovic-Vitanovic, V. (1996). Ecological study of the Obedska Bara (in Serbian). Belgrade: Publ. Research Centre "Srbijasume".

Martinovic-Vitanovic, V. \& Kalafatic V. (1995). The basic hydrobiological characteristics of inland waters in Yugoslavia (in Serbian). In V. Stevanovic \& V. Vasic (Eds.), The Biodiversity of Yugoslavia with a Survey of Species of International Significance (pp. 97-115). Belgrade: Publ. Fac. Biol., Univ. Belgrade, and Ecolibri.

Martinovic-Vitanovic, V. \& Kalafatic V. (1999). The evolution of Obedska Bara - a swamp in the floodplain of the River Sava. Contributions to the Zoogeography and Ecology of the Eastern Mediterranean Region 1, 359-365.

Martinovic-Vitanovic, V., \& Kalafatic, V. (2002). Limnological investigations of the Danube in Yugoslavia. Report of the Joint Investigations of the Danube River on the Territory of the FR Yugoslavia within the International JDS-ITR Program. Publ. Rep. of Serbia, Belgrade: Ministry for Protection of Natural Resources and Environment, Federal Hydrometeorol. Inst.

Martinovic-Vitanovic, V., \& Kalafatic, V. (2006). Limnological study of Lake Srebrno (in Serbian). In the Project Implementation of the EU Water Framework Directive in Serbia - Definition of the typology and reference conditions for the standing water. The case study of Lake Srebrno in August and October 2006. Belgrade: Jaroslav Cerni Institute for Development of Water Resources, Belgrade and Ministry of Agriculture, Forestry and Water Management of the Republic of Serbia - Water Directorate.

Martinovic-Vitanovic, V., Jakovcev-Todorovic, D. \& Kalafatic V. (2006). Qualitative study of the River Danube (river kilometre 1433-845.6), with special emphasis on the oligochaetes. Arch. Hydrobiol. Suppl., 158 (3)-Large Rivers, 16(3), 427-452.

Martinovic-Vitanovic, V., Obradovic, S., Milankov, M. \& Kalafatic V. (2008). Bottom fauna of the Sava River (r-km 61.5-0.5) in Serbia. Arch. Hydrobiol. Suppl., 166 (1-2)-Large Rivers, 18(1-2), 209-241.

Martinovic-Vitanovic, V., Milankov, V. \& Kalafatic V. (2009/2010). First record of freshwater bryozoans (Bryozoa: Phylactolaemata) in the aquatic invertebrate fauna of Serbia. Limnologica, 40(1), 73-81. DOI:10.1016/j.limno.2009.04.001.

Moog, O.(Ed.) (1995). Fauna aquatica Austriaca. Katalog zur autökologischen Einstufung aquatischer Organismen Österreichs. Teil III, B, Metazoa. Wien: Bundesministerium f. Land- u. Fortwirtschaft.

Moog, O. (Ed.) (2002). Fauna aquatica Austriaca. Wasserwirtschaftskataster. Wien: Bundesministerium f. Land- u. Fortwirtschaft.

Nguyen, H.L., Leermakers, M., Osán, J., Török, S. \& Baeyens W. (2005). Heavy metals in Lake Balaton: water column, suspended matter, sediment and biota. Sci. Total Environ., 340, 213-230. DOI:10.1016/j.scitotenv.2004.07.032. 
Obolewski, K., Glinska-Lewezuk, K. \& Kobus S. (2009). Effect of hydrological connectivity on the molluscan community structure in oxbow lakes of the Lyna river. Oceanol. Hydrobiol Stud., 38(4), 75-88. DOI:10.2478/ v10009-009-0045-1.

OECD (1982). Eutrophication of water, monitoring, assessment and control. Paris: Organization for Economic Cooperation and Development.

The Act on Water Classification of Inter-republican, International and Coastal Waters of Yugoslavia (in Serbian). (1978). The Official Gazette of the SFR of Yugoslavia 6/78.

Watercourses Classification Act (in Serbian). (1968). The Official Gazette of the SR of Serbia 5/68.

Pantle, R. \& Buck H. (1955). Die biologische Überwachung der Gewässer und die Darstellung der Ergebnisse. Gas-Wasserfach, 96, 604-620.

Reckendorfer, W., Baranyi, C., Funk, A. \& Schiemer F. (2006). Floodplain restoration by reinforcing hydrological connectivity: expected effects on aquatic mollusc communities. J. Appl. Ecol., 43, 474-484. DOI: 10.1111/j.1365-2664.2006.01155.x.

Resh V.H. \& Rosenberg D.M. (1984). The ecology of aquatic isects. New York: Praeger Publishers, CBS Inc.

Risnoveanu, G. \& Vadineanu A. (2002). Observations on the population dynamics of Potamothrix hammoniensis (Michaelsen, 1901) (Tubificidae, Oligochaeta) in lake Isacova in the Danube Delta. Hydrobiologia, 479, $23-30$. DOI:10.1023/A:1021012111295.

Risnoveanu, G. \& Vadineanu A. (2003). Long-term functional changes within the Oligochaeta communities within the Danube River Delta, Romania. Hydrobiologia, 506-509, 399-405. DOI:10.1023/B:HYDR.0000008597.03953.89.

Ristola, T. (2002). Assessment of sediment toxicity using the midge Chironomus riparius (Diptera: Chironomidae). $\mathrm{PhD}$ Dissertation in Biology: 5, University of Joensuu, Finland.

Rosenberg D.M. \& Resh V.H. (Eds.) (1993). Freshwater biomonitoring and benthic macroinvertebrates. New York: Chapman and Hall.

Selesi, Dj. (1979). Beiträge zur Bestimmung der trophischen Stufe einiger Flachen Gewässer in Woiwodina. In Second Congress of Ecologists of Yugoslavia (pp. 1945-1951). Zagreb: Publ. Association of Ecological Societies of Yugoslavia.

Solimini, A.G., Free, G., Donahue, I., Irvine, K., Pusch, M., Rossaro, B., Sandin, L., \& Cardoso, A.C. (2006). Using benthic macroinvertebrates to assess ecological status of lakes; current knowledge and way forward to support WFD implementation. Brussels: European Commission. Directorate-General. Joint Research Centre, Institute for Environment and Sustainability. (EUR $22347 \mathrm{EN}$ )

Sørensen, T. (1948). A method of establishing groups of equal amplitude in plant sociology based on similarity of species content and its application to analyses of the vegetation on Danish commons. Kobenhavn: Det Kongel. Danske Videnskabernes Selskab Biol. Skrifter V.

Specziar, A. \& Biro P. (1998). Spatial distribution and short-term changes of benthic macrofauna in Lake Balaton (Hungary). Hydrobiologia, 389, 203-216. DOI:10.1023/A:1003563228162.

Statsoft, Inc. (1997). Statistica for Windows (Computer program manual). Tulsa, OK, USA: Statsoft, Inc.

Timm, T. (1999). A guide to the Estonian Annelida. Tartu/Tallinn: Eston. Academy Publ.

Thornton, K. W., Kimmel B. L. \& Payne F. E. (Eds.) (1990). Reservoir limnology: ecological perspectives. New Jersey: John Wiley \& Sons.

USEPA. (2002). A guidance manual to support the assessment of contaminated sediments in freshwater ecosystems. Volume III - Interpretations of the results of sediment quality investigations. Chicago: United States of America Environmental Protection Agency.

Verdonschot, P.F.M. (1999). Micro-distribution of oligochaetes in a soft-bottomed lowland stream (Elsbeek; the Netherlands). Hydrobiologia, 406, 149-163. DOI:10.1023/A:1003796403364.

Wentworth, C.K. (1922). A scale of grade and class terms for clastic sediments. J. Geol., 30, 377_392.

Wiederholm, T. (Ed.) (1983). Chironomidae of the Holarctic region. Keys and diagnoses. Part 1. Larvae. Entomol. Scand., Suppl.19, 1-457.

Appendix

Abbreviations of recorded and analyzed species of the Lake Srebrno benthic fauna:

N-NEMATODA, O1-Limnodrilus claparedeanus, O2-Limnodrilus hoffmeisteri, O3-Limnodrilus sp., O4-Potamothrix hammoniensis, Of-Oligochaeta fragments, Hi-Glossiphonia complanata, Hy-HYDRACARINA, B-BRYOZOA, Ch1-Chironomus plumosus, Ch2-Chironomus riparius, Ch3-Chironomus sp., Ch4-Cryptochironomus defectus, Ch5-Dicrotendipes nervosus, Ch6-Einfeldia carbonaria, Ch7-Glyptotendipes pallens, Ch8-Polypedilum nubeculosum, Ch9-Procladius Skuse, Cg-Ceratopogonidae, Chb-Chaoborus sp., T-Philopotamus montanus 